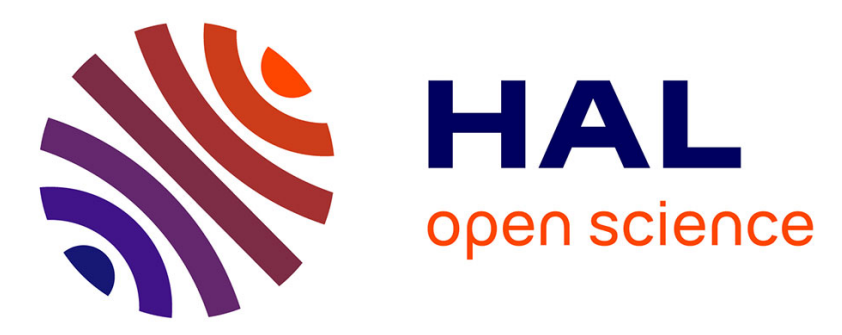

\title{
A Graph-Theoretic Approach to Fault Detection and Isolation for Structured Bilinear Systems
}

Taha Boukhobza, Frédéric Hamelin, Sébastien Canitrot

\section{To cite this version:}

Taha Boukhobza, Frédéric Hamelin, Sébastien Canitrot. A Graph-Theoretic Approach to Fault Detection and Isolation for Structured Bilinear Systems. International Journal of Control, 2008, 81 (4), pp.661-678. 10.1080/00207170701630396 . hal-00312019

\section{HAL Id: hal-00312019 https://hal.science/hal-00312019}

Submitted on 25 Aug 2008

HAL is a multi-disciplinary open access archive for the deposit and dissemination of scientific research documents, whether they are published or not. The documents may come from teaching and research institutions in France or abroad, or from public or private research centers.
L'archive ouverte pluridisciplinaire $\mathbf{H A L}$, est destinée au dépôt et à la diffusion de documents scientifiques de niveau recherche, publiés ou non, émanant des établissements d'enseignement et de recherche français ou étrangers, des laboratoires publics ou privés. 


\title{
A graph-theoretic approach to fault detection and isolation for structured bilinear systems
}

\author{
T. Boukhobza* $\dagger$, F. Hamelin $\dagger$ and S. Canitrot $\dagger$ \\ $\dagger$ Centre de Recherche en Automatique de Nancy (CRAN), Nancy University, CNRS UMR 7039,BP 239, 54506 Vandoeuvre \\ Cedex, Nancy, France. \\ (Received 00 Month 200x; in final form 00 Month 200x)
}

\begin{abstract}
Using a graph-theoretic approach, we address in this paper some issues related to the fault detection and isolation for structured bilinear systems. Considering a structured bilinear system submitted to faults and disturbances, we give necessary and sufficient conditions to the solvability of the so-called bilinear fundamental problem of residual generation. We treat as well the cases where the system is submitted to multiple failures occurring simultaneously or only one at a time. One of the main advantages of the proposed analysis tool is that all the given conditions are easy to check because they deal with finding paths in a digraph. This makes our approach well suited to study large scale systems.
\end{abstract}

\section{Introduction}

The fault detection and isolation (FDI) problem has received considerable attention in the two last decades (Blanke et al. 2003, Gertler 1998). In fact, the detection and the location of incipient faults is important for safety critical systems where a malfunction can cause human and material damages. An FDI system is mainly made of two parts. The first one is related to the generation and the evaluation of some signals called residuals which must be sensitive to faults occurring on the system. The second part concerns the decision-making part on the basis of these residuals. In this paper, we concentrate on the residual generation part in the particular case of bilinear systems.

The class of bilinear systems (BLS), representing the particular nonlinear systems whose dynamics are jointly linear in the state and the input variables, was introduced in control theory in the 1960's. Industrial process control, economics and biology (switched circuits, mechanical brakes, controlled suspension systems, immunological systems, population growth, enzyme kinetics, ... ) provide examples of BLS. For these reasons, many works deal with BLS. The problem of FDI for BLS has been tackled mainly since the works of (Yu and Shields 1996, Yang and Saif 1995). Authors of (Yang and Saif 1995) explore the design of a reduced order observer with unknown inputs for estimating the fault values. Results of (Yu and Shields 1996, Yu et al. 1996) are based on unknown input observers and sufficient conditions for the existence of bilinear FDI observers are given. In (Kinnaert 1999), the author proposes two robust FDI schemes for BLS which are not necessarily affine in the failure modes. Finally, one of the most important results is given in (Hammouri et al. 2001). Using a geometric approach, the authors provide necessary and sufficient conditions for the solvability of the so-called bilinear fundamental problem of residual generation (BFPRG). This problem extends the fundamental problem of residual generation (FPRG) defined and solved for linear systems in (Massoumnia et al. 1989). The solvability conditions of such a problem for nonlinear systems, which are affine in the control inputs and in the failure modes, have been also given in (Hammouri et al. 1999, De Persis and Isidori 2001) using the concept of observability codistributions (De Persis and Isidori 2000).

${ }^{*}$ Corresponding author. Email: taha.boukhobza@cran.uhp-nancy.fr

International Journal of Control 
Up to now, the solvability conditions of the BFPRG are mainly based on algebraic or geometric tools. Nevertheless, the use of such methods assumes the exact knowledge of the state space matrices characterizing the system's model. In many modeling problems, these matrices have a number of fixed zero entries determined by the physical structure while the remaining entries are not precisely known. To study the properties of the systems for which we have a poor knowledge, the idea is that we only keep the zero/nonzero entries in the state space matrices. Thus, we consider models where the fixed zeros are conserved while the nonzero entries are replaced by free parameters. There is a huge amount of interesting works using this kind of models called structured models because their study requires a low computational burden which allows to deal with large scale systems.

Many studies on structured systems are related to the graph-theoretic approach. Until now, this approach is principally dedicated to linear systems for which structural properties, such as controllability, observability, solvability of several classical control problems including disturbance rejection, input-output decoupling, fault detection and isolation, are studied. Survey paper (Dion et al. 2003) reviews the most significant results in this area. From these works, it results that the graph-theoretic approach provides simple and elegant solutions and so is very well suited to analyse large scale systems. Unfortunately, few works based on graph-theoretic methods deal with nonlinear systems.

Among these works, we can cite (Düstgör et al. 2004, Staroswiecki 2002) where the authors use bipartite graphs to analyse the structural fault detectability and isolability for linear and nonlinear systems. Nevertheless, in this approach, the graphic representation of the following linear system $\left(\Sigma_{1}\right)$ and bilinear one $\left(\Sigma_{2}\right)$ are similar.

$$
\Sigma_{1}:\left\{\begin{array}{l}
\dot{x}_{1}=\lambda_{1} f_{1} \\
\dot{x}_{2}=\lambda_{2} x_{1}+\lambda_{3} u_{1}+\lambda_{4} f_{2} \\
\dot{x}_{3}=\lambda_{5} x_{1} \\
y_{1}=\lambda_{6} x_{3}, \\
y_{2}=\lambda_{7} x_{2}
\end{array} \Sigma_{2}:\left\{\begin{array}{l}
\dot{x}_{1}=\lambda_{1} f_{1} \\
\dot{x}_{2}=\lambda_{2} u_{1} x_{1}+\lambda_{3} f_{2} \\
\dot{x}_{3}=\lambda_{4} x_{1} \\
y_{1}=\lambda_{5} x_{3} \\
y_{2}=\lambda_{6} x_{2}
\end{array}=A_{0} x+u_{1} A_{1} x+E_{1} f_{1}+E_{2} f_{2}\right.\right.
$$

Therefore, the conditions provided in (Düstgör et al. 2004) lead to the same conclusion: for both systems $\left(\Sigma_{1}\right)$ and $\left(\Sigma_{2}\right)$ the faults are detectable and isolable. Nevertheless, using the results of (Hammouri et al. 2001, De Persis and Isidori 2001), we can prove that for almost all the values of $\lambda_{i}$ the BFPRG is not solvable for bilinear system $\left(\Sigma_{2}\right)$. Indeed, the bipartite graphs used in (Düstgör et al. 2004, Staroswiecki 2002) are not so complete to express all the nonlinearities particularly the product $u \cdot x$.

The purpose of this paper is to determine the conditions for the solvability of some residual generation problems for structured bilinear systems (SBLS) using a graph-theoretic approach. More precisely, we study the BFPRG with multiple failure events. We consider the case where the system is submitted to multiple and possibly simultaneous failures events and the case where only one failure can occur at a time on the system.

The paper is organised as follows: after Section 2, which is devoted to the problem formulation, a digraph representation of SBLS is defined in Section 3. The necessary and sufficient conditions for the solvability of BFPRG are enounced in Section 4. Finally, some concluding remarks are made. 


\section{Problem statement}

In this paper, we address the generic solvability of the Bilinear Fundamental Problem of Residual Generation (BFPRG) for SBLS:

$$
\left(\Sigma_{\Lambda}\right):\left\{\begin{array}{l}
\dot{x}=A_{0} x+\sum_{i=1}^{m} u_{i} A_{i} x+E f+H w \\
y=C x
\end{array}\right.
$$

where $x \in \mathbb{R}^{n}, u \in \mathbb{R}^{m}, f \in \mathbb{R}^{q}, w \in \mathbb{R}^{d}$ and $y \in \mathbb{R}^{p}$ are respectively the state, the input, the fault, the disturbance and the output vectors. $A_{i}$, for $i=0, \ldots, m, C, E$, and $H$ represent matrices of appropriate dimensions, which elements are either fixed to zero or assumed free nonzero parameters. We can parameterize these nonzero entries by scalar real (nonzero) parameters $\lambda_{i}, i=1, \ldots, h$ forming a parameter vector $\Lambda=\left(\lambda_{1}, \lambda_{2}, \ldots, \lambda_{h}\right)^{T} \in \mathbb{R}^{h}$. If all the nonzero parameters $\lambda_{i}$ are fixed, we obtain an admissible realization of structured system $\left(\Sigma_{\Lambda}\right)$. Theoretic properties of each realization can be studied according to the values of $\lambda_{i}$. We say that a property is true generically if it is true for almost all the realizations of structured system $\left(\Sigma_{\Lambda}\right)$. Here, "for almost all the realizations" is to be understood (Dion et al. 2003, van der Woude 2000) as "for all parameter values $\left(\Lambda \in \mathbb{R}^{h}\right)$ except for those in some proper algebraic variety in the parameter space". The proper algebraic variety for which the property is not true is the zero set of some nontrivial polynomial with real coefficients in the system parameters $\lambda_{1}, \lambda_{2}, \ldots, \lambda_{h}$ or, equivalently, it is an algebraic variety, which has Lebesgue measure zero.

Consider SBLS $\left(\Sigma_{\Lambda}\right)$, we define the BFPRG as the problem of finding filter of the form (2):

$$
\left\{\begin{array}{l}
\dot{z}=\phi(z, y)+\psi(z, y) u \\
r=h(z, y)
\end{array}\right.
$$

where residual $r \in \mathbb{R}^{q}$ is such that, for all $i \in\{1,2, \ldots, q\}$

i. when $f_{i}=0, r_{i}$ is insensitive to $w$ and to all $f_{j}$ for $j \neq i$. It must decay to zero for all admissible inputs $u$ and whatever $x(0), z(0)$;

ii. $r_{i}$ is affected by $f_{i}$ i.e. it takes a non-zero value for at least some $t \geq 0$ whatever $x(0)$ and $z(0)$.

We can relax the constraints on the residual generation problem solvability in the case of multiple failure modes by assuming that there is only one failure present at a time. We define the Problem of Residual Generation with No Simultaneous Faults as the problem of finding filter (2), where residual $r \in \mathbb{R}^{k}$, (with $2^{k}-1 \geq q$ ), is such that

i. when no failure is present (and only in this case), all the residual components $r_{i}$ decay asymptotically to zero whatever the value of $w$, for all admissible inputs $u$ and for all initial conditions $x(0)$ and $z(0)$;

ii. in the $i^{\text {th }}$ failure mode (i.e. $f_{i} \neq 0$ and $f_{\ell}=0, \forall \ell \neq i$ ), residual $r_{j}$ for $j \in \Upsilon_{i} \subseteq\{1,2, \ldots, k\}$ are nonzero while other residuals $r_{j}$ for $j \in\{1,2, \ldots, k\} \backslash \Upsilon_{i}$ decay to zero for all admissible inputs $u$, for all initial conditions $x(0)$, $z(0)$ and for all $w(t)$. Here, the prespecified family of coding sets $\Upsilon_{i}$ is chosen such that $\forall i \neq j, \Upsilon_{i} \neq \Upsilon_{j}$ in order to uniquely identify each failure. 
The BFPRG has been treated and solved in (Hammouri et al. 2001) for non structured systems using a geometric approach. By a straightforward extension of the results enounced in (Hammouri et al. 2001) to SBLS, we can deduce that, in the single fault case $(q=1)$, the BFPRG has generically a solution iff for almost all the realizations of system (1), $E \notin \bar{O}_{H}$, where $\bar{O}_{H}$ is the minimal unobservability subspace containing $\operatorname{Im}(H)$. The objective of this paper is to find graphic conditions equivalent to the previous one. It turns out that these graphic conditions are easier to check and so more adapted to tackle large scale systems with unknown parameters. Indeed, the computation of $\bar{O}_{H}$ is quite complicated in the case where we deal with large scale systems: $\bar{O}_{H}$ is the limit of the sequence $\left\{\begin{array}{l}T_{0}=\mathcal{R}_{H}+\operatorname{ker} C \\ T_{i+1}=\mathcal{R}_{H}+\left(\bigcap_{j=0}^{m}\left(A_{j}^{-1} T_{i}\right)\right) \cap \operatorname{ker} C\end{array}\right.$

where $\mathcal{R}_{H}$ represents the minimum invariant $\left(C-\left(A_{0}, \ldots, A_{m}\right)\right)$ subspace including $\operatorname{Im}(H)$. More precisely, $\mathcal{R}_{H}$ is the limit of the following non-decreasing sequence:

$$
\left\{\begin{array}{l}
\mathcal{R}_{0}=\operatorname{Im}(H), \\
\mathcal{R}_{i+1}=\mathcal{R}_{i}+\sum_{j=0}^{m} A_{j}\left(\mathcal{R}_{i} \cap \operatorname{ker} C\right)
\end{array}\right.
$$

The computation of these sequences is characterized by a high computational burden, particularly concerning the term $\left(\bigcap_{j=0}^{m}\left(A_{j}^{-1} T_{i}\right)\right) \cap \operatorname{ker} C$ when the parameters of the system are not fixed. One of the main objectives of our method is to provide simpler conditions from a computational point of view. Indeed, one of the advantages of graph-theoretic approach is precisely the computational simplicity of the obtained results. Indeed, we recall that for linear systems, the graphic solvability conditions of the FPRG are quite simple and are given in (Commault et al. 2002).

Our aim is to obtain a solvability criterion for the BFPRG as simple as the one described above for linear systems. To do so, we focus on the minimum invariant $\left(C-\left(A_{0}, \ldots, A_{m}\right)\right)$ subspace including $\operatorname{Im}(H)$ noted above by $\mathcal{R}_{H}$. More precisely, our aim is to quantify the generic dimension of $C \mathcal{R}_{H}$ noted $g_{-} \operatorname{dim}\left(C \mathcal{R}_{H}\right)$ and not to characterize $\mathcal{R}_{H}$ since the latter varies with the specific values of parameters $\lambda_{i}$.

The importance of $g_{-} \operatorname{dim}\left(C \mathcal{R}_{H}\right)$ for BFPRG solvability is highlighted in the following lemma:

Lemma 2.1 Consider structured bilinear system $\left(\Sigma_{\Lambda}\right)$, in the single fault case i.e. $q=1$, the BFPRG has generically a solution iff

$$
g_{-} \operatorname{dim}\left(C \mathcal{R}_{H, E}\right)>g_{-} \operatorname{dim}\left(C \mathcal{R}_{H}\right)
$$

where $\mathcal{R}_{H, E}$ represents the minimum invariant $\left(C-\left(A_{0}, \ldots, A_{m}\right)\right)$ subspace including $\operatorname{Im}(H)+\operatorname{Im}(E)$.

\section{Proof:}

Sufficiency: Relation (4) implies that $\mathcal{R}_{H, E} \nsubseteq \mathcal{R}_{H}$ and particularly that there exists generically a vector $u \notin \operatorname{ker} C$ such that $u \in \mathcal{R}_{H, E}$ and $C u \notin C \mathcal{R}_{H}$. Thus, $u \notin \mathcal{R}_{H}+\operatorname{ker} C$ and so, as $\bar{O}_{H} \subseteq \mathcal{R}_{H}+\operatorname{ker} C, u \notin \bar{O}_{H}$. Nevertheless, since $u \in \mathcal{R}_{H, E}$, we have that $u \in \bar{O}_{H, E}$ and so $\bar{O}_{H, E} \nsubseteq \bar{O}_{H} \Rightarrow E \notin \bar{O}_{H}$, which means that the BFPRG is generically solvable.

Necessity: If the BFPRG has not generically a solution then $E \in \bar{O}_{H}$. This implies that $\bar{O}_{H, E}=\bar{O}_{H}$ and so $\overline{C \bar{O}_{H, E}}=C \bar{O}_{H}$. Nevertheless, since $\mathcal{R}_{H, E} \subseteq \bar{O}_{H, E} \subseteq \mathcal{R}_{H, E}+\operatorname{ker} C$, we have that $C \mathcal{R}_{H, E} \subseteq C \bar{O}_{H, E} \subseteq C\left(\mathcal{R}_{H, E}+\operatorname{ker} C\right)$. 
Thus, $C \bar{O}_{H, E}=C \mathcal{R}_{H, E}$ and similarly $C \bar{O}_{H}=C \mathcal{R}_{H}$. Therefore, we have that $C \bar{O}_{H, E}=C \bar{O}_{H} \Rightarrow g_{-} \operatorname{dim}\left(C \mathcal{R}_{H, E}\right)=$ $g_{-} \operatorname{dim}\left(C \mathcal{R}_{H}\right)$.

The approach we propose is based on the previous Lemma and so a great part of the paper deal with the computation of the generic dimension of the subspace $C \mathcal{R}_{H}$ using a graph approach. In the next section, we introduce the main graphic tools which allow us to analyse the BFPRG solvability.

\section{Graphic representation of structured bilinear systems}

In this section, we firstly define the digraph, which can be associated to SBLS $\left(\Sigma_{\Lambda}\right)$ in order to study the BFPRG generic solvability. Next, we give some useful notations and definitions.

\subsection{Digraph definition for structured bilinear systems}

The digraph associated to $\left(\Sigma_{\Lambda}\right)$ is noted $\mathcal{G}\left(\Sigma_{\Lambda}\right)$ and is constituted by a vertex set $\mathcal{V}$ and an edge set $\mathcal{E}: \mathcal{G}\left(\Sigma_{\Lambda}\right)=$ $(\mathcal{V}, \mathcal{E})$. The vertices are associated to the state, output, disturbance and fault components of $\left(\Sigma_{\Lambda}\right)$ and the directed edges represent links between these variables.

More precisely, $\mathcal{V}=\mathbf{X} \cup \mathbf{Y} \cup \mathbf{F} \cup \mathbf{W}$, where $\mathbf{X}=\left\{\mathbf{x}_{\mathbf{1}}, \ldots, \mathbf{x}_{\mathbf{n}}\right\}$ is the set of state vertices, $\mathbf{Y}=\left\{\mathbf{y}_{1}, \ldots, \mathbf{y}_{\mathbf{p}}\right\}$ is the set of output vertices, $\mathbf{F}=\left\{\mathbf{f}_{\mathbf{1}}, \ldots, \mathbf{f}_{\mathbf{q}}\right\}$ is the set of fault vertices and $\mathbf{W}=\left\{\mathbf{w}_{\mathbf{1}}, \ldots, \mathbf{w}_{\mathbf{d}}\right\}$ is the set of disturbance vertices.

The edge set is $\mathcal{E}=\bigcup_{k=0}^{m} A_{k}$-edges $\cup C$-edges $\cup E$-edges $\cup H$-edges, where for $k=0, \ldots, m, A_{k}$-edges $=$ $\left\{\left(\mathbf{x}_{\mathbf{i}}, \mathbf{x}_{\mathbf{j}}\right) \mid A_{k}(j, i) \neq 0\right\}, C$-edges $=\left\{\left(\mathbf{x}_{\mathbf{i}}, \mathbf{y}_{\mathbf{j}}\right) \mid C(j, i) \neq 0\right\}, \quad E$-edges $=\left\{\left(\mathbf{f}_{\mathbf{i}}, \mathbf{x}_{\mathbf{j}}\right) \mid E(j, i) \neq 0\right\}$ and $H$-edges $=$ $\left\{\left(\mathbf{w}_{\mathbf{i}}, \mathbf{x}_{\mathbf{j}}\right) \mid H(j, i) \neq 0\right\}$.

Here, $\left(\mathbf{v}_{\mathbf{1}}, \mathbf{v}_{\mathbf{2}}\right)$ denotes a directed edge from vertex $\mathbf{v}_{\mathbf{1}} \in \mathcal{V}$ to vertex $\mathbf{v}_{\mathbf{2}} \in \mathcal{V}$. We indicate the indice $u_{i}$ over each $A_{i}$-edges and $u_{0}$ also under $C$-edges, $E$-edges and $H$-edges. Moreover, we take the following notations: $\bar{A}_{0}$-edges $=A_{0}$-edges $\cup C$-edges $\cup E$-edges $\cup H$-edges and for $i=1, \ldots, m, \bar{A}_{i}$-edges $=A_{i}$-edges .

Hereafter, we illustrate our proposed digraph representation with a simple example.

Example 3.1 Consider a structured bilinear system defined by:

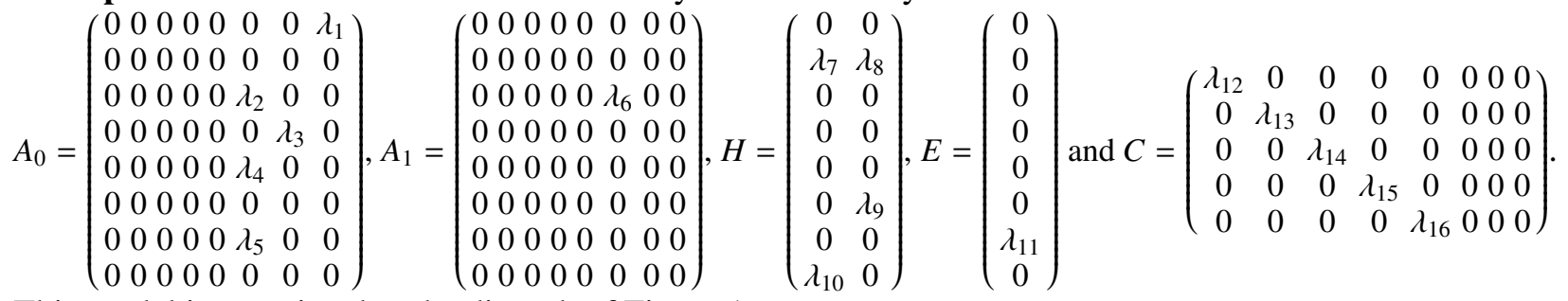

This model is associated to the digraph of Figure 1.

Remark 1 For the sake of simplicity in the presentation, we will assume that matrix $C$ is on the form $C=$ $\left(\operatorname{diag}\left(\lambda_{i_{1}}, \lambda_{i_{2}}, \ldots, \lambda_{i_{p}}\right) \mid \mathbf{0}\right)$. The notations used in this paper can be easily modified to take any matrix $C$ into account. 


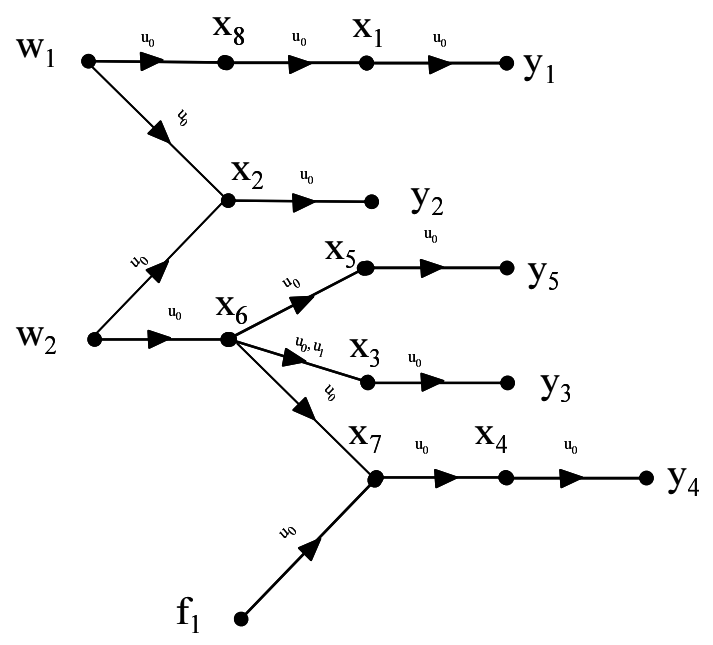

Figure 1.: Digraph associated to system of Example 3.1

\subsection{Definitions and notations}

- $P=\mathbf{v}_{\mathbf{0}} \stackrel{u_{i_{1}}}{\longrightarrow} \mathbf{v}_{\mathbf{1}} \stackrel{u_{i_{2}}}{\longrightarrow} \ldots \stackrel{u_{i_{s}}}{\longrightarrow} \mathbf{v}_{\mathbf{s}}$ denotes a path $P$, which contains vertices $\mathbf{v}_{\mathbf{0}}, \mathbf{v}_{\mathbf{1}}, \ldots, \mathbf{v}_{\mathbf{s}}$ and where $\left(\mathbf{v}_{\mathbf{j}-\mathbf{1}}, \mathbf{v}_{\mathbf{j}}\right) \in \bar{A}_{i_{j}}$-edges, for $j=1, \ldots, s$. The length of a path is the number of edges that the path uses, counting multiple edges multiple times. For each path $P$, we denote by $\alpha(P)$ the end vertex of $P$. $P$ is said an $\mathbf{Y}$-topped path iff $\alpha(P) \in \mathbf{Y}$.

- Some paths are disjoint if they have no common vertex.

- Let $\mathcal{V}_{1}$ be a vertex subset, $\operatorname{card}\left(\mathcal{V}_{1}\right)$ represents the cardinality of $\mathcal{V}_{1}$.

- Let $\mathcal{V}_{1}$ and $\mathcal{V}_{2}$ denote two vertex subsets. Path $P$ is said a $\mathcal{V}_{1}-\mathcal{V}_{2}$ path if its begin vertex belongs to $\mathcal{V}_{1}$ and its end vertex belongs to $\mathcal{V}_{2}$.

- A set of $l$ disjoint $\mathcal{V}_{1}-\mathcal{V}_{2}$ paths is called a $\mathcal{V}_{1}-\mathcal{V}_{2}$ linking of size $l$. The linkings, which consist of a maximal number of disjoint $\mathcal{V}_{1}-\mathcal{V}_{2}$ paths are called maximal $\mathcal{V}_{1}-\mathcal{V}_{2}$ linkings. We define $\rho\left(\mathcal{V}_{1}, \mathcal{V}_{2}\right)$ as the maximal number of disjoint $\mathcal{V}_{1}-\mathcal{V}_{2}$ paths. Consider a $\mathcal{V}_{1}-\mathcal{V}_{2}$ linking noted $\mathcal{L}_{0}$, we define $\alpha\left(\mathcal{L}_{0}\right) \stackrel{\text { def }}{=} \bigcup_{P \in \mathcal{L}_{0}}\{\alpha(P)\}$. Finally, we denote by $\mu\left(\mathcal{V}_{1}, \mathcal{V}_{2}\right)$ the minimal number of vertices of $\mathbf{X} \cup \mathbf{Y}$ belonging to a maximal $\mathcal{V}_{1}-\mathcal{V}_{2}$ linking.

In example 3.1, $\rho(\mathbf{W} \cup \mathbf{F}, \mathbf{Y})=3$. There exist seven maximal $\mathbf{W} \cup \mathbf{F}$-Y linkings: $\mathcal{L}_{1}=\left\{\mathbf{f}_{\mathbf{1}} \stackrel{u_{0}}{\longrightarrow} \mathbf{x}_{\mathbf{7}} \stackrel{u_{0}}{\longrightarrow} \mathbf{x}_{\mathbf{4}} \stackrel{u_{0}}{\longrightarrow}\right.$ $\left.\mathbf{y}_{\mathbf{4}}, \mathbf{w}_{\mathbf{1}} \stackrel{u_{0}}{\longrightarrow} \mathbf{x}_{\mathbf{8}} \stackrel{u_{0}}{\longrightarrow} \mathbf{x}_{\mathbf{1}} \stackrel{u_{0}}{\longrightarrow} \mathbf{y}_{\mathbf{1}}, \mathbf{w}_{\mathbf{2}} \stackrel{u_{0}}{\longrightarrow} \mathbf{x}_{\mathbf{2}} \stackrel{u_{0}}{\longrightarrow} \mathbf{y}_{\mathbf{2}}\right\}, \mathcal{L}_{2}=\left\{\mathbf{f}_{\mathbf{1}} \stackrel{u_{0}}{\longrightarrow} \mathbf{x}_{\mathbf{7}} \stackrel{u_{0}}{\longrightarrow} \mathbf{x}_{\mathbf{4}} \stackrel{u_{0}}{\longrightarrow} \mathbf{y}_{\mathbf{4}}, \mathbf{w}_{\mathbf{1}} \stackrel{u_{0}}{\longrightarrow} \mathbf{x}_{\mathbf{8}} \stackrel{u_{0}}{\longrightarrow} \mathbf{x}_{\mathbf{1}} \stackrel{u_{0}}{\longrightarrow}\right.$ $\left.\mathbf{y}_{\mathbf{1}}, \mathbf{w}_{\mathbf{2}} \stackrel{u_{0}}{\longrightarrow} \mathbf{x}_{\mathbf{6}} \stackrel{u_{0}}{\longrightarrow} \mathbf{x}_{\mathbf{5}} \stackrel{u_{0}}{\longrightarrow} \mathbf{y}_{\mathbf{5}}\right\}, \mathcal{L}_{3}=\left\{\mathbf{f}_{\mathbf{1}} \stackrel{u_{0}}{\longrightarrow} \mathbf{x}_{7} \stackrel{u_{0}}{\longrightarrow} \mathbf{x}_{\mathbf{4}} \stackrel{u_{0}}{\longrightarrow} \mathbf{y}_{\mathbf{4}}, \mathbf{w}_{\mathbf{1}} \stackrel{u_{0}}{\longrightarrow} \mathbf{x}_{\mathbf{8}} \stackrel{u_{0}}{\longrightarrow} \mathbf{x}_{\mathbf{1}} \stackrel{u_{0}}{\longrightarrow} \mathbf{y}_{\mathbf{1}}, \mathbf{x}_{\mathbf{2}} \stackrel{u_{0}}{\longrightarrow} \mathbf{x}_{\mathbf{6}} \stackrel{u_{0}}{\longrightarrow}\right.$ $\left.\mathbf{x}_{\mathbf{3}} \stackrel{u_{0}}{\longrightarrow} \mathbf{y}_{\mathbf{3}}\right\}, \mathcal{L}_{4}=\left\{\mathbf{f}_{\mathbf{1}} \stackrel{u_{0}}{\longrightarrow} \mathbf{x}_{\mathbf{7}} \stackrel{u_{0}}{\longrightarrow} \mathbf{x}_{\mathbf{4}} \stackrel{u_{0}}{\longrightarrow} \mathbf{y}_{\mathbf{4}}, \mathbf{w}_{\mathbf{1}} \stackrel{u_{0}}{\longrightarrow} \mathbf{x}_{\mathbf{8}} \stackrel{u_{0}}{\longrightarrow} \mathbf{x}_{\mathbf{1}} \stackrel{u_{0}}{\longrightarrow} \mathbf{y}_{\mathbf{1}}, \mathbf{w}_{\mathbf{2}} \stackrel{u_{0}}{\longrightarrow} \mathbf{x}_{\mathbf{6}} \stackrel{u_{1}}{\longrightarrow} \mathbf{x}_{\mathbf{3}} \stackrel{u_{0}}{\longrightarrow} \mathbf{y}_{\mathbf{3}}\right\}$, $\mathcal{L}_{5}=\left\{\mathbf{f}_{\mathbf{1}} \stackrel{u_{0}}{\longrightarrow} \mathbf{x}_{7} \stackrel{u_{0}}{\longrightarrow} \mathbf{x}_{\mathbf{4}} \stackrel{u_{0}}{\longrightarrow} \mathbf{y}_{\mathbf{4}}, \mathbf{w}_{\mathbf{1}} \stackrel{u_{0}}{\longrightarrow} \mathbf{x}_{\mathbf{2}} \stackrel{u_{0}}{\longrightarrow} \mathbf{y}_{\mathbf{2}}, \mathbf{w}_{\mathbf{2}} \stackrel{u_{0}}{\longrightarrow} \mathbf{x}_{\mathbf{6}} \stackrel{u_{0}}{\longrightarrow} \mathbf{x}_{\mathbf{5}} \stackrel{u_{0}}{\longrightarrow} \mathbf{y}_{\mathbf{5}}\right\}, \mathcal{L}_{6}=\left\{\mathbf{f}_{\mathbf{1}} \stackrel{u_{0}}{\longrightarrow} \mathbf{x}_{7} \stackrel{u_{0}}{\longrightarrow} \mathbf{x}_{\mathbf{4}} \stackrel{u_{0}}{\longrightarrow}\right.$ $\left.\mathbf{y}_{\mathbf{4}}, \mathbf{w}_{\mathbf{1}} \stackrel{u_{0}}{\longrightarrow} \mathbf{x}_{\mathbf{2}} \stackrel{u_{0}}{\longrightarrow} \mathbf{y}_{\mathbf{2}}, \mathbf{w}_{\mathbf{2}} \stackrel{u_{0}}{\longrightarrow} \mathbf{x}_{\mathbf{6}} \stackrel{u_{0}}{\longrightarrow} \mathbf{x}_{\mathbf{3}} \stackrel{u_{0}}{\longrightarrow} \mathbf{y}_{\mathbf{3}}\right\}, \mathcal{L}_{7}=\left\{\mathbf{f}_{\mathbf{1}} \stackrel{u_{0}}{\longrightarrow} \mathbf{x}_{\mathbf{7}} \stackrel{u_{0}}{\longrightarrow} \mathbf{x}_{\mathbf{4}} \stackrel{u_{0}}{\longrightarrow} \mathbf{y}_{\mathbf{4}}, \mathbf{w}_{\mathbf{1}} \stackrel{u_{0}}{\longrightarrow} \mathbf{x}_{\mathbf{2}} \stackrel{u_{0}}{\longrightarrow} \mathbf{y}_{\mathbf{2}}, \mathbf{w}_{\mathbf{2}} \stackrel{u_{0}}{\longrightarrow}\right.$ $\left.\mathbf{x}_{\mathbf{6}} \stackrel{u_{1}}{\longrightarrow} \mathbf{x}_{\mathbf{3}} \stackrel{u_{0}}{\longrightarrow} \mathbf{y}_{\mathbf{3}}\right\}$. Moreover, $\mu(\mathbf{W} \cup \mathbf{F}, \mathbf{Y})=8$.

- Consider a vertex subset $\mathcal{V}_{0}$, we say that path $P=\mathbf{v}_{\mathbf{0}} \stackrel{u_{i_{1}}}{\longrightarrow} \mathbf{v}_{\mathbf{1}} \stackrel{u_{i_{2}}}{\longrightarrow} \ldots \stackrel{u_{i_{s}}}{\longrightarrow} \mathbf{v}_{\mathbf{s}}$ is included in $\mathcal{V}_{0}$ if $\left\{\mathbf{v}_{1}, \ldots, \mathbf{v}_{\mathrm{s}-1}\right\} \subseteq \mathcal{V}_{0}$.

- To any path $P=\mathbf{v}_{\mathbf{0}} \stackrel{u_{i_{1}}}{\longrightarrow} \mathbf{v}_{\mathbf{1}} \stackrel{u_{i_{2}}}{\longrightarrow} \ldots \stackrel{u_{i_{s}}}{\longrightarrow} \mathbf{v}_{\mathbf{s}}$, we associate an unique indice noted $\sigma(P)$ and defined by the 
ordered monomial $u_{i_{1}} \cdot u_{i_{2}} \cdot \ldots \cdot u_{i_{s}}$. A path $P$ is uniquely characterized by its indice and the vertices it covers. Note that, two paths are distinct when they have distinct indices, even if they cover the same vertices.

In Example 3.1, paths $P_{1}=\mathbf{w}_{\mathbf{2}} \stackrel{u_{0}}{\longrightarrow} \mathbf{x}_{\mathbf{6}} \stackrel{u_{0}}{\longrightarrow} \mathbf{x}_{\mathbf{3}}$ and $P_{2}=\mathbf{w}_{\mathbf{2}} \stackrel{u_{0}}{\longrightarrow} \mathbf{x}_{\mathbf{6}} \stackrel{u_{1}}{\longrightarrow} \mathbf{x}_{\mathbf{3}}$, which include the same vertices, are distinct because they have distinct indices. Indeed, $\sigma\left(P_{1}\right)=u_{0} \cdot u_{0}$ and $\sigma\left(P_{2}\right)=u_{0} \cdot u_{1}$.

- The indices are ordered monomials in $u_{0}, u_{1}, \ldots, u_{m}$ of the form $u_{i_{1}} \cdot u_{i_{2}} \cdot \ldots \cdot u_{i_{\ell}}$, where $\left\{i_{1}, i_{2}, \ldots, i_{\ell}\right\} \subseteq\{0, \ldots, m\}$ are not necessarily distinct. The set of all possible ordered monomials is noted $\wp(u)$. To present the properties of $\wp(u)$ provided with a binary noncommutative operation noted ".", let us consider three indices $\sigma_{1}=u_{i_{1}} \cdot u_{i_{2}} \cdot \ldots \cdot u_{i_{\ell}} \in \wp(u)$, $\sigma_{2}=u_{j_{1}} \cdot u_{j_{2}} \cdot \ldots \cdot u_{j_{k}} \in \wp(u)$ and $\sigma_{3} \in \wp(u)$, we have:

$\diamond \sigma_{1}=\sigma_{2}$ iff $\ell=k$ and $i_{1}=j_{1}, i_{2}=j_{2}, \ldots, i_{\ell}=j_{\ell}$;

$\diamond \sigma_{1} \cdot \sigma_{2}=u_{i_{1}} \cdot u_{i_{2}} \cdot \ldots \cdot u_{i_{\ell}} \cdot u_{j_{1}} \cdot u_{j_{2}} \cdot \ldots \cdot u_{j_{k}}$

$\diamond \sigma_{1} \cdot\left(\sigma_{2} \cdot \sigma_{3}\right)=\left(\sigma_{1} \cdot \sigma_{2}\right) \cdot \sigma_{3}=\sigma_{1} \cdot \sigma_{2} \cdot \sigma_{3}$;

$\diamond$ For operation ".", 1 represents the indice of the zero length path. It is the identity element and for any indice $\sigma$, we have $1 \cdot \sigma=\sigma \cdot 1=\sigma$;

$\diamond$ The length of an indice consists in the number of terms $u_{i}$ it contains, counting multiple terms $u_{i}$ multiple times.

- Paths $P_{1}=\mathbf{v}_{\mathbf{0}}^{\mathbf{1}} \stackrel{u_{i_{1}}}{\longrightarrow} \mathbf{v}_{\mathbf{1}}^{\mathbf{1}} \stackrel{u_{i_{2}}}{\longrightarrow} \cdots \stackrel{u_{i_{1}}}{\longrightarrow} \mathbf{v}_{\mathbf{k}_{1}}^{\mathbf{1}}$ and $P_{2}=\mathbf{v}_{\mathbf{0}}^{\mathbf{2}} \stackrel{u_{j_{1}}}{\longrightarrow} \mathbf{v}_{\mathbf{1}}^{\mathbf{2}} \stackrel{u_{j_{2}}}{\longrightarrow} \cdots \stackrel{u_{j_{k_{2}}}}{\longrightarrow} \mathbf{v}_{\mathbf{k}_{2}}^{\mathbf{2}}$ are $A$-disjoint iff the following conditions hold

Cond1- $\mathbf{v}_{\mathbf{k}_{1}}^{1} \neq \mathbf{v}_{\mathbf{k}_{2}}^{2}$;

Cond2- $\forall j=1,2, \ldots, \min \left(k_{1}, k_{2}\right)$ such that $\mathbf{v}_{\mathbf{k}_{1}-\mathbf{j}}^{\mathbf{1}}=\mathbf{v}_{\mathbf{k}_{2}-\mathbf{j}}^{\mathbf{2}}$, sub-paths $\mathbf{v}_{\mathbf{k}_{1}-\mathbf{j}}^{\mathbf{1}} \stackrel{u_{i_{1}-j+1}}{\longrightarrow} \cdots \stackrel{u_{i_{k_{1}}}}{\longrightarrow} \mathbf{v}_{\mathbf{k}_{\mathbf{1}}}^{\mathbf{1}}$ and $\mathbf{v}_{\mathbf{k}_{2}-\mathbf{j}}^{\mathbf{2}} \stackrel{u_{j_{k_{2}}-j+1}}{\longrightarrow} \cdots \stackrel{u_{j_{k_{2}}}}{\longrightarrow} \mathbf{v}_{\mathbf{k}_{2}}^{\mathbf{2}}$ must have distinct indices.

In Example 3.1, $P_{1}=\mathbf{w}_{2} \stackrel{u_{0}}{\longrightarrow} \mathbf{x}_{\mathbf{6}} \stackrel{u_{1}}{\longrightarrow} \mathbf{x}_{\mathbf{3}}$ and $P_{2}=\mathbf{w}_{2} \stackrel{u_{0}}{\longrightarrow} \mathbf{x}_{\mathbf{6}} \stackrel{u_{0}}{\longrightarrow} \mathbf{x}_{7}$ are $A$-disjoint whereas neither $P_{1}$ and $P_{3}=\mathbf{w}_{2} \stackrel{u_{0}}{\longrightarrow} \mathbf{x}_{\mathbf{6}} \stackrel{u_{0}}{\longrightarrow} \mathbf{x}_{\mathbf{3}}$ nor $P_{2}$ and $P_{3}$ are $A$-disjoint.

- Some paths are $A$-disjoint if they are mutually $A$-disjoint. Note that two disjoint paths are naturally $A$-disjoint.

- A set of $l A$-disjoint $\mathcal{V}_{1}-\mathcal{V}_{2}$ paths is called a $\mathcal{V}_{1}-\mathcal{V}_{2} A$-disjoint linking of size $l$. The linkings, which consist on a maximal number of $A$-disjoint paths, are called maximal $\mathcal{V}_{1}-\mathcal{V}_{2} A$-disjoint linkings.

- Consider two $\mathcal{V}$-X paths $P_{1}=\mathbf{v}_{\mathbf{0}}^{\mathbf{1}} \stackrel{u_{i_{1}}}{\longrightarrow} \mathbf{v}_{\mathbf{1}}^{\mathbf{1}} \stackrel{u_{i_{2}}}{\longrightarrow} \cdots \stackrel{u_{i_{k_{1}}}}{\longrightarrow} \mathbf{v}_{\mathbf{k}_{1}}^{\mathbf{1}}$ and $P_{2}=\mathbf{v}_{\mathbf{0}}^{\mathbf{2}} \stackrel{u_{j_{1}}}{\longrightarrow} \mathbf{v}_{\mathbf{1}}^{\mathbf{2}} \stackrel{u_{j_{2}}}{\longrightarrow} \cdots \stackrel{u_{j_{k_{2}}}}{\longrightarrow} \mathbf{v}_{\mathbf{k}_{2}}^{\mathbf{2}}$. We say that $P_{1}$ is "multiple" of $P_{2}$, if there exists $j \leq k_{1}$ such that $\mathbf{v}_{\mathbf{0}}^{\mathbf{1}} \stackrel{u_{i_{1}}}{\longrightarrow} \mathbf{v}_{\mathbf{1}}^{\mathbf{1}} \stackrel{u_{i_{2}}}{\longrightarrow} \cdots \stackrel{u_{i_{j}}}{\longrightarrow} \mathbf{v}_{\mathbf{j}}^{\mathbf{1}}$ and $P_{2}$ are not $A$-disjoint. In this case, if $j_{0}$ is the minimal value of $j>0$ such that $\mathbf{v}_{\mathbf{0}}^{\mathbf{1}} \stackrel{u_{i_{1}}}{\longrightarrow} \mathbf{v}_{\mathbf{1}}^{\mathbf{1}} \stackrel{u_{i_{2}}}{\longrightarrow} \cdots \stackrel{u_{i_{j}}}{\longrightarrow} \mathbf{v}_{\mathbf{j}}^{\mathbf{1}}$ and $P_{2}$ are not $A$-disjoint, then we denote by $\sigma\left(P_{1} / P_{2}\right)$ the indice $\sigma\left(\mathbf{v}_{\mathbf{j}_{0}}^{\mathbf{1}} \stackrel{u_{i_{j_{0}+1}}}{\longrightarrow} \cdots \stackrel{u_{i_{k_{1}}}}{\longrightarrow} \mathbf{v}_{\mathbf{k}_{1}}^{\mathbf{1}}\right)=u_{i_{j_{0}+1}} \cdot \ldots \cdot u_{i_{k_{1}}}$ and we use the notation $P_{1} \equiv P_{2} \odot u_{i_{j_{0}+1}} \cdot u_{i_{j_{0}+2}} \cdot \ldots \cdot u_{i_{k_{1}}}$. If $P_{1}$ and $P_{2}$ are not $A$-disjoint then we note $\sigma\left(P_{1} / P_{2}\right)=1$ and $P_{1} \equiv P_{2}$.

We say that $P_{1}$ is a "strict multiple" of $P_{2}$ when $P_{1}$ is a "multiple" of $P_{2}$ and $\sigma\left(P_{1} / P_{2}\right) \neq 1$.

In Example $1, P_{4}=\mathbf{w}_{1} \stackrel{u_{0}}{\longrightarrow} \mathbf{x}_{\mathbf{8}} \stackrel{u_{0}}{\longrightarrow} \mathbf{x}_{\mathbf{1}}$ is multiple of $P_{5}=\mathbf{w}_{\mathbf{1}} \stackrel{u_{0}}{\longrightarrow} \mathbf{x}_{\mathbf{2}}$ and we write $P_{4} \equiv P_{5} \odot u_{0}$.

Note that if $P_{1} \equiv P_{2} \odot \sigma_{1}, P_{2} \equiv P_{3}$ and $P_{4} \equiv P_{3} \odot \sigma_{1}$ then we do not have necessarily that $P_{1} \equiv P_{4}$ neither that $P_{1} \equiv P_{3} \odot \sigma_{1}$.

- Consider vertex subsets $\mathbf{W}_{\mathbf{0}} \subseteq \mathbf{W} \cup \mathbf{F}$ and $\boldsymbol{\Gamma}_{\mathbf{i n t}} \subseteq \mathbf{X}_{\mathbf{C}} \stackrel{\text { def }}{=}\left\{\mathbf{x}_{\mathbf{1}}, \mathbf{x}_{\mathbf{2}}, \ldots, \mathbf{x}_{\mathbf{p}}\right\}$. Due to Remark $1, \mathbf{X}_{\mathbf{C}}$ represents the set of all state vertices, which have edges towards $\mathbf{Y}$ and are such that $\mathbf{x}_{\mathbf{i}}$ is linked to $\mathbf{y}_{\mathbf{i}}$ for $i=1, \ldots, p$.

A path $Q_{1}$ is said prime $\mathbf{W}_{\mathbf{0}}-\boldsymbol{\Gamma}_{\text {int }}$ path if $Q_{1}$ is a $\mathbf{W}_{\mathbf{0}}-\boldsymbol{\Gamma}_{\text {int }}$ path and there does not exist a $\mathbf{W}_{\mathbf{0}}-\boldsymbol{\Gamma}_{\text {int }}$ path $P$ such that $Q_{1}$ 
is a strict multiple of $P$.

In Example 3.1, $Q_{1}=\mathbf{w}_{\mathbf{1}} \stackrel{u_{0}}{\longrightarrow} \mathbf{x}_{\mathbf{2}}$ and $Q_{2}=\mathbf{w}_{\mathbf{2}} \stackrel{u_{0}}{\longrightarrow} \mathbf{x}_{\mathbf{2}}$ are prime $\mathbf{W}-\mathbf{X}_{\mathbf{C}}$ paths, whereas $\mathbf{w}_{\mathbf{2}} \stackrel{u_{0}}{\longrightarrow} \mathbf{x}_{\mathbf{6}} \stackrel{u_{1}}{\longrightarrow} \mathbf{x}_{\mathbf{3}}$ and $\mathbf{w}_{\mathbf{1}} \stackrel{u_{0}}{\longrightarrow} \mathbf{x}_{\mathbf{8}} \stackrel{u_{0}}{\longrightarrow} \mathbf{x}_{\mathbf{1}}$ are not prime $\mathbf{W}-\mathbf{X}_{\mathbf{C}}$ paths.

- To each structured matrix $B \in \mathbb{R}^{\ell \times n}$, we can associate a bipartite graph $\mathcal{B}_{\mathcal{M}}=\left(\mathcal{V}^{+} ; \mathcal{V}^{-} ; \mathcal{E}_{\mathcal{M}}\right)$, where $\mathcal{V}^{+} \stackrel{\text { def }}{=}\left\{\mathbf{x}_{\mathbf{1}}^{+}, \mathbf{x}_{\mathbf{2}}^{+}, \ldots, \mathbf{x}_{\ell}^{+}\right\}, \mathcal{V}^{-} \stackrel{\text { def }}{=} \mathbf{X}$ and $\mathcal{E}_{\mathcal{M}} \stackrel{\text { def }}{=}\left\{\left(\mathbf{x}_{\mathbf{i}}^{+}, \mathbf{x}_{\mathbf{j}}\right), B(j, i) \neq 0\right\}$. Let $\theta\left(\mathcal{V}_{1}, \mathcal{V}_{2}\right)$ denote the maximal number of disjoint edges from $\mathcal{V}_{1} \subseteq \mathcal{V}^{+}$to $\mathcal{V}_{2} \subseteq \mathcal{V}^{-}$. We define the notion of maximal matching partition of matrix $B$ as $\mathcal{M}(B)=\left\{\gamma \subseteq \mathbf{X}, \operatorname{card}(\gamma)=\theta\left(\mathcal{V}^{+}, \mathbf{X}\right)=\theta\left(\mathcal{V}^{+}, \gamma\right)\right\}$. Note that $\theta\left(\mathcal{V}^{+}, \mathbf{X}\right)$ represents the maximal matching of matrix $B$ and it is equal to the generic rank of matrix $B$, noted $g_{-} \operatorname{rank}(B)$ (Murota 1987).

In Example 3.1, $\mathcal{M}\left(A_{0}\right)=\left\{\left\{\mathbf{x}_{1}, \mathbf{x}_{\mathbf{3}}, \mathbf{x}_{\mathbf{4}}\right\},\left\{\mathbf{x}_{\mathbf{1}}, \mathbf{x}_{\mathbf{5}}, \mathbf{x}_{\mathbf{4}}\right\},\left\{\mathbf{x}_{\mathbf{1}}, \mathbf{x}_{\mathbf{7}}, \mathbf{x}_{\mathbf{4}}\right\}\right\}, \mathcal{M}\left(A_{1}\right)=\left\{\left\{\mathbf{x}_{\mathbf{3}}\right\}\right\}, \mathcal{M}(H)=$ $\left\{\left\{\mathbf{x}_{\mathbf{2}}, \mathbf{x}_{\mathbf{8}}\right\},\left\{\mathbf{x}_{\mathbf{2}}, \mathbf{x}_{\mathbf{6}}\right\},\left\{\mathbf{x}_{\mathbf{6}}, \mathbf{x}_{\mathbf{8}}\right\}\right\}$ and $\mathcal{M}(C)=\left\{\left\{\mathbf{x}_{1}, \mathbf{x}_{\mathbf{2}}, \mathbf{x}_{\mathbf{3}}, \mathbf{x}_{\mathbf{4}}, \mathbf{x}_{\mathbf{5}}\right\}\right\}$.

\section{Main results}

First, we recall the result obtained for linear systems in (Commault et al. 2002), in order to highlight the simplicity of the conditions obtained using graphic tools:

THEOREM 4.1 Consider a structured linear system (i.e. $\left(\Sigma_{\Lambda}\right)$, with matrices $A_{i}=0, \forall i \geq 1$ ) represented by digraph $\mathcal{G}\left(\Sigma_{\Lambda}\right)$. The FPRG is generically solvable iff $\rho(\mathbf{W} \cup \mathbf{F}, \mathbf{Y})=\operatorname{card}(\mathbf{F})+\rho(\mathbf{W}, \mathbf{Y})$

Our aim is to obtain, for the BFPRG generic solvability, graphic conditions which are as simple as the one given in Theorem 4.1. First, let us do the following assumption on the system:

A1: Every vertex in $\mathbf{X} \cup \mathbf{F} \cup \mathbf{W}$ is the begin vertex of an $\mathbf{Y}$-topped path.

We can motivate assumption $\mathbf{A 1}$ by the fact that if a vertex of $\mathbf{F}$ does not satisfy $\mathbf{A 1}$, then this fault component is obviously not detectable and we can conclude immediately that the BFPRG is not solvable. So, we are interested only in the cases where whole elements of $\mathbf{F}$ satisfy A1. Moreover, if there exist elements of $\mathbf{X} \cup \mathbf{W}$ which do not verify A1, we can, without loss of generality, remove these vertices as they have no effect on the BFPRG solvability.

\subsection{Bilinear Fundamental Problem of Residual Generation with a single fault}

For the sake of simplicity, we begin in this subsection by the single fault case i.e. $q=1$, and so $E \in R^{n \times 1}$ and $\mathbf{F}=\left\{\mathbf{f}_{\mathbf{1}}\right\}$. Hereafter, we give some definitions useful to the graphical characterization of $g_{-} \operatorname{dim}\left(C \mathcal{R}_{H}\right)$ for bilinear systems.

$\bullet \mathbf{J}_{\mathbf{1}} \stackrel{\text { def }}{=}\left\{\mathbf{x}_{\mathbf{j}} \in \mathbf{X}, \rho\left(\mathbf{W}, \mathbf{Y} \cup\left\{\mathbf{x}_{\mathbf{j}}\right\}\right)=\rho(\mathbf{W}, \mathbf{Y})\right.$ and $\left.\mu\left(\mathbf{W}, \mathbf{Y} \cup\left\{\mathbf{x}_{\mathbf{j}}\right\}\right)=\mu(\mathbf{W}, \mathbf{Y})\right\}$.

It is obvious that, for a structured system which is parameterized by $\Lambda=\left(\lambda_{1}, \lambda_{2}, \ldots, \lambda_{h}\right)^{T} \in \mathbb{R}^{h}, \mathcal{R}_{H}$ varies with the specific values of the parameters $\lambda_{i}$, but there exists a constant subspace that includes $\mathcal{R}_{H}$ for almost any value of the parameters(Commault et al. 1997). This subspace is called the minimal structured fixed subspace containing $\mathcal{R}_{H}$ and is noted $\mathcal{R}_{H}^{f}$. For structured linear systems $\left(A_{1}, \ldots, A_{m}=0\right)$, when Assumption $\mathbf{A 1}$ is satisfied, it is shown that(Commault et al. 1997)

$$
\mathcal{R}_{H}^{f}=\operatorname{span}\left\{e_{j}, \mathbf{x}_{\mathbf{j}} \in \mathbf{X} \backslash \mathbf{J}_{\mathbf{1}}\right\}
$$


where $e_{j}$ is the $j^{\text {th }}$ Euclidean vector.

It is important to note that, including the eventual zero length paths in the linkings, we can rewrite relation (5) using the maximal matching partition of matrix $H$ :

$\mathbf{J}_{\mathbf{1}} \stackrel{\text { def }}{=}\left\{\mathbf{x}_{\mathbf{j}} \in \mathbf{X}, \max _{\gamma \in \mathbf{\Omega}_{\mathbf{0}}} \rho\left(\gamma, \mathbf{Y} \cup\left\{\mathbf{x}_{\mathbf{j}}\right\}\right)=\rho_{0}\right.$ and $\left.\min _{\gamma \in \mathbf{\Omega}_{\mathbf{0}}, \rho\left(\gamma, \mathbf{Y} \cup\left\{\mathbf{x}_{\mathbf{j}}\right\}\right)=\rho_{0}} \mu\left(\gamma, \mathbf{Y} \cup\left\{\mathbf{x}_{\mathbf{j}}\right\}\right)=\mu_{0}\right\}$,

where $\boldsymbol{\Omega}_{\mathbf{0}}=\mathcal{M}(H), \rho_{0}=\max _{\gamma \in \mathbf{\Omega}_{\mathbf{0}}} \rho(\gamma, \mathbf{Y})$ and $\mu_{0}=\min _{\gamma \in \mathbf{\Omega}_{\mathbf{0}}, \rho(\gamma, \mathbf{Y})=\rho_{0}} \mu(\gamma, \mathbf{Y})$.

- $\boldsymbol{\Delta}_{\mathbf{1}} \stackrel{\text { def }}{=}\left\{\mathbf{x}_{\mathbf{j}} \in \mathbf{X} \mid \max _{\gamma \in \mathbf{\Omega}_{\mathbf{0}}} \rho\left(\gamma, \mathbf{Y} \cup\left\{\mathbf{x}_{\mathbf{j}}\right\}\right)>\rho_{0}\right.$ or $\left.\min _{\gamma \in \mathbf{\Omega}_{\mathbf{0}}, \rho\left(\gamma, \mathbf{Y} \cup\left\{\mathbf{x}_{\mathbf{j}}\right\}\right)=\rho_{0}} \mu\left(\gamma, \mathbf{Y} \cup\left\{\mathbf{x}_{\mathbf{j}}\right\}\right)<\mu_{0}-1\right\}$. Obviously, $\boldsymbol{\Delta}_{\mathbf{1}} \subseteq \mathbf{X} \backslash \mathbf{J}_{\mathbf{1}}$.

- $\boldsymbol{\Gamma}_{\mathbf{1}} \stackrel{\text { def }}{=} \mathbf{X} \backslash\left(\mathbf{J}_{\mathbf{1}} \cup \boldsymbol{\Delta}_{\mathbf{1}}\right)$.

We have partitioned $\mathbf{X} \backslash \mathbf{J}_{\mathbf{1}}$ into two subsets $\boldsymbol{\Delta}_{\mathbf{1}}$ and $\boldsymbol{\Gamma}_{\mathbf{1}}$. $\boldsymbol{\Delta}_{\mathbf{1}}$ does not contain any vertex of $\mathbf{X}_{\mathbf{C}}$. Thus, due to the particular structure of matrix $C, \rho(\mathbf{W}, \mathbf{Y})=\rho\left(\mathbf{W}, \mathbf{X}_{\mathbf{c}}\right)=\rho\left(\mathbf{W}, \boldsymbol{\Gamma}_{1}\right)$ and $C e_{\Delta}=0$ where $e_{\Delta}$ is a diagonal $n \times n$ matrix such that $e_{\Delta}(i, i)=\left\{\begin{array}{ll}1 & \text { if } \mathbf{x}_{\mathbf{i}} \in \Delta_{\mathbf{1}} \\ 0 & \text { otherwise }\end{array}\right.$. Consequently and according to (5), for linear systems, we can easily prove that $C \mathcal{R}_{H}=C e_{\Gamma} \mathcal{R}_{H}$ and $g_{-} \operatorname{dim}\left(C \mathcal{R}_{H}\right)=g_{-} \operatorname{dim}\left(C e_{\Gamma} \mathcal{R}_{H}\right)=g_{-} \operatorname{dim}\left(e_{\Gamma} \mathcal{R}_{H}\right)$ where $e_{\Gamma}$ is a diagonal $n \times n$ matrix such that $e_{\Gamma}(i, i)=\left\{\begin{array}{ll}1 & \text { if } \mathbf{x}_{\mathbf{i}} \in \boldsymbol{\Gamma}_{\mathbf{1}} \\ 0 & \text { otherwise }\end{array}\right.$.

- We can subdivise $\boldsymbol{\Gamma}_{\mathbf{1}}$ into two subsets:

$\boldsymbol{\Gamma}_{\text {int }} \stackrel{\text { def }}{=}\left\{\mathbf{x}_{\mathbf{i}} \in \boldsymbol{\Gamma}_{\mathbf{1}}, \exists \mathbf{W}-\boldsymbol{\Gamma}_{\mathbf{1}}\right.$ paths $P$ and $Q$ included in $\boldsymbol{\Delta}_{\mathbf{1}}$ such that $\alpha(Q)=\mathbf{x}_{\mathbf{i}}$ and $P$ is a strict multiple of $\left.\mathrm{Q}\right\}$, $\boldsymbol{\Gamma}_{\text {ext }}=\boldsymbol{\Gamma}_{\mathbf{1}} \backslash \boldsymbol{\Gamma}_{\text {int }}$. Obviously $\boldsymbol{\Gamma}_{\text {int }} \subseteq \mathbf{X}_{\mathbf{C}}$.

In Example 3.1, we have that $\rho(\mathbf{W}, \mathbf{Y})=2=\rho_{0}, \mu(\mathbf{W}, \mathbf{Y})=5=\mu_{0}$. On the one hand, $\forall \mathbf{x}_{\mathbf{i}} \in \mathbf{X}, \rho\left(\mathbf{W}, \mathbf{Y} \cup\left\{\mathbf{x}_{\mathbf{i}}\right\}\right)=2$. On the other hand, $\forall \mathbf{x}_{\mathbf{i}} \in \mathbf{X} \backslash\left\{\mathbf{x}_{\mathbf{4}}\right\}, \mu\left(\mathbf{W}, \mathbf{Y} \cup\left\{\mathbf{x}_{\mathbf{i}}\right\}\right)<5$ and only $\mu\left(\mathbf{W}, \mathbf{Y} \cup\left\{\mathbf{x}_{\mathbf{4}}\right\}\right)=5$. That implies $\mathbf{J}_{\mathbf{1}}=\left\{\mathbf{x}_{\mathbf{4}}\right\}$. Since, $\mathbf{x}_{\mathbf{6}}$ and $\mathbf{x}_{\mathbf{8}}$ are the only state vertices which satisfy $\mu\left(\mathbf{W}, \mathbf{Y} \cup\left\{\mathbf{x}_{\mathbf{j}}\right\}\right)=3<5-1$, then $\Delta_{\mathbf{1}}=\left\{\mathbf{x}_{\mathbf{6}}, \mathbf{x}_{\mathbf{8}}\right\}$. Therefore, $\boldsymbol{\Gamma}_{\mathbf{1}}=\left\{\mathbf{x}_{\mathbf{1}}, \mathbf{x}_{\mathbf{2}}, \mathbf{x}_{\mathbf{3}}, \mathbf{x}_{\mathbf{5}}, \mathbf{x}_{\mathbf{7}}\right\}$. Since there exists one $\mathbf{W}-\boldsymbol{\Gamma}_{\mathbf{1}}$ path $P_{1}=\mathbf{w}_{\mathbf{1}} \stackrel{u_{0}}{\longrightarrow} \mathbf{x}_{\mathbf{8}} \stackrel{u_{0}}{\longrightarrow} \mathbf{x}_{\mathbf{1}}$ included in $\boldsymbol{\Delta}_{\mathbf{1}}$, which is strict multiple of the $\mathbf{W}-\boldsymbol{\Gamma}_{\mathbf{1}}$ path $Q_{1}=\mathbf{w}_{\mathbf{1}} \stackrel{u_{0}}{\longrightarrow} \mathbf{x}_{\mathbf{2}}$, we have that $\boldsymbol{\Gamma}_{\mathrm{int}}=\left\{\mathbf{x}_{\mathbf{2}}\right\}$.

- Let us consider a $\mathbf{W}-\boldsymbol{\Gamma}_{\text {ext }}$ path $P_{2}$, a prime $\mathbf{W}-\boldsymbol{\Gamma}_{\text {int }}$ path $Q_{1}$ and a monomial $\sigma_{1}$. We say that $Q_{1} \odot \sigma_{1}$ is a strict multiple of path $P_{2}$ if $P_{2}$ is multiple of $Q_{1}$ and if there exists a monomial $\sigma_{1}^{\prime} \neq 1$ such that $\sigma_{1}=\sigma\left(P_{2} / Q_{1}\right) \cdot \sigma_{1}^{\prime}$.

To introduce the next definitions, let us now compute $\mathcal{R}_{H}$ for Example 3.1:

$-\mathcal{R}_{0}=\operatorname{span}\left\{\left(\begin{array}{c}0 \\ \lambda_{7} \\ 0 \\ 0 \\ 0 \\ 0 \\ 0 \\ \lambda_{10}\end{array}\right),\left(\begin{array}{c}0 \\ \lambda_{8} \\ 0 \\ 0 \\ 0 \\ \lambda_{9} \\ 0 \\ 0\end{array}\right)\right\}, g_{-} \operatorname{dim}\left(\mathcal{R}_{0}\right)=2$ and $g_{-} \operatorname{dim}\left(\mathcal{R}_{0} \cap \operatorname{ker} C\right)=1$. Indeed, a combination of the two vectors constituting $\mathcal{R}_{0}$ is included in $\operatorname{ker} C: \mathcal{R}_{0} \cap \operatorname{ker} C=\operatorname{span}\left\{\left(\begin{array}{c}0 \\ 0 \\ 0 \\ 0 \\ 0 \\ -\lambda_{7} \lambda_{9} \\ \lambda_{8} \lambda_{10}\end{array}\right)\right\}$. $-\mathcal{R}_{1}=\operatorname{span}\left\{\left(\begin{array}{c}0 \\ \lambda_{7} \\ 0 \\ 0 \\ 0 \\ 0 \\ 0 \\ \lambda_{10}\end{array}\right),\left(\begin{array}{c}0 \\ \lambda_{8} \\ 0 \\ 0 \\ 0 \\ \lambda_{9} \\ 0 \\ 0\end{array}\right),\left(\begin{array}{c}0 \\ 0 \\ -\lambda_{6} \lambda_{\lambda} \lambda_{9} \\ 0 \\ 0 \\ 0 \\ 0 \\ 0\end{array}\right)\left(\begin{array}{c}\lambda_{1} \lambda_{8} \lambda_{10} \\ 0 \\ 0 \\ 0 \\ -\lambda_{4} \lambda_{0} \lambda_{9} \\ -\lambda_{5} \lambda_{\lambda_{9}} \lambda_{9} \\ 0\end{array}\right)\right\} \cdot g_{-} \operatorname{dim}\left(\mathcal{R}_{1}\right)=4$ and $g_{-} \operatorname{dim}\left(\mathcal{R}_{1} \cap \operatorname{ker} C\right)=1=g_{-} \operatorname{dim}\left(\mathcal{R}_{0} \cap \operatorname{ker} C\right)$. Thus, $\mathcal{R}_{H}=\mathcal{R}_{1}$. Consequently, $C \mathcal{R}_{H}=$ $\operatorname{span}\left\{\left(\begin{array}{c}0 \\ \lambda_{13} \lambda_{7} \\ 0 \\ 0 \\ 0\end{array}\right),\left(\begin{array}{c}\lambda_{13} \lambda_{8} \\ 0 \\ 0 \\ 0\end{array}\right),\left(\begin{array}{c}0 \\ 0 \\ -\lambda_{14} \lambda_{6} \lambda_{7} \lambda_{9} \\ 0 \\ 0\end{array}\right)\left(\begin{array}{c}\lambda_{12} \lambda_{1} \lambda_{8} \lambda_{10} \\ 0 \\ 0 \\ 0 \\ -\lambda_{16} \lambda_{4} \lambda_{7} \lambda_{9}\end{array}\right)\right\}$ and $g_{-} \operatorname{dim}\left(C \mathcal{R}_{H}\right)=3$

There exists obviously a matrix $B$ such that $C \mathcal{R}_{H}=\operatorname{Im}(B)$. Generically, $\mathcal{M}(B)=\left\{\left\{\mathbf{x}_{\mathbf{1}}, \mathbf{x}_{\mathbf{2}}, \mathbf{x}_{\mathbf{3}}\right\},\left\{\mathbf{x}_{\mathbf{2}}, \mathbf{x}_{\mathbf{3}}, \mathbf{x}_{\mathbf{5}}\right\}\right\}$. Graphi- 
cally, $\mathcal{R}_{H}$ can be traduced using $\mathbf{W}-\boldsymbol{\Gamma}_{\mathbf{1}} A$-disjoint linkings: $\mathcal{L}_{1}=\left\{\mathbf{w}_{\mathbf{1}} \stackrel{u_{0}}{\longrightarrow} \mathbf{x}_{\mathbf{8}} \stackrel{u_{0}}{\longrightarrow} \mathbf{x}_{\mathbf{1}}, \mathbf{w}_{\mathbf{2}} \stackrel{u_{0}}{\longrightarrow} \mathbf{x}_{\mathbf{2}}, \mathbf{w}_{\mathbf{2}} \stackrel{u_{0}}{\longrightarrow} \mathbf{x}_{\mathbf{6}} \stackrel{u_{1}}{\longrightarrow} \mathbf{x}_{\mathbf{3}}\right\}$ and $\mathcal{L}_{2}=\left\{\mathbf{w}_{\mathbf{2}} \stackrel{u_{0}}{\longrightarrow} \mathbf{x}_{\mathbf{6}} \stackrel{u_{0}}{\longrightarrow} \mathbf{x}_{\mathbf{5}}, \mathbf{w}_{\mathbf{2}} \stackrel{u_{0}}{\longrightarrow} \mathbf{x}_{\mathbf{2}}, \mathbf{w}_{\mathbf{2}} \stackrel{u_{0}}{\longrightarrow} \mathbf{x}_{\mathbf{6}} \stackrel{u_{1}}{\longrightarrow} \mathbf{x}_{\mathbf{3}}\right\}$. Nevertheless, linking $\mathcal{L}_{3}=\left\{\mathbf{w}_{\mathbf{1}} \stackrel{u_{0}}{\longrightarrow} \mathbf{x}_{\mathbf{8}} \stackrel{u_{0}}{\longrightarrow}\right.$ $\left.\mathbf{x}_{\mathbf{1}}, \mathbf{w}_{\mathbf{2}} \stackrel{u_{0}}{\longrightarrow} \mathbf{x}_{\mathbf{6}} \stackrel{u_{0}}{\longrightarrow} \mathbf{x}_{\mathbf{5}}, \mathbf{w}_{\mathbf{2}} \stackrel{u_{0}}{\longrightarrow} \mathbf{x}_{\mathbf{2}}, \mathbf{w}_{\mathbf{2}} \stackrel{u_{0}}{\longrightarrow} \mathbf{x}_{\mathbf{6}} \stackrel{u_{1}}{\longrightarrow} \mathbf{x}_{\mathbf{3}}\right\}$ is also an $A$-disjoint linking but cannot be associated to $\mathcal{R}_{H}$. Indeed, even if $P=\mathbf{w}_{\mathbf{1}} \stackrel{u_{0}}{\longrightarrow} \mathbf{x}_{\mathbf{8}} \stackrel{u_{0}}{\longrightarrow} \mathbf{x}_{\mathbf{1}}$ and $P^{\prime}=\mathbf{w}_{\mathbf{2}} \stackrel{u_{0}}{\longrightarrow} \mathbf{x}_{\mathbf{6}} \stackrel{u_{0}}{\longrightarrow} \mathbf{x}_{5}$ are $A$-disjoint, they are "linked" by the fact that $P \equiv Q_{1} \odot u_{0}$ and $P^{\prime} \equiv Q_{2} \odot u_{0}$ where $Q_{1}=\mathbf{w}_{\mathbf{1}} \stackrel{u_{0}}{\longrightarrow} \mathbf{x}_{\mathbf{2}}$ and $Q_{2}=\mathbf{w}_{\mathbf{2}} \stackrel{u_{0}}{\longrightarrow} \mathbf{x}_{\mathbf{2}}$ are prime $\mathbf{W}-\boldsymbol{\Gamma}_{\text {int }}$ paths which have same end vertex $\mathbf{x}_{\mathbf{2}}$.

To take into account the link between $P$ and $P^{\prime}$, which is not explicitly apparent in the digraph associated to the system, we define the notion of complete family. Intuitively, a $\mathbf{W}-\boldsymbol{\Gamma}_{\mathbf{1}}$ path $\mathbf{w}_{\mathbf{i}} \stackrel{u_{0}}{\longrightarrow} \mathbf{x}_{\mathbf{j}_{1}} \stackrel{u_{i_{1}}}{\longrightarrow} \ldots \stackrel{u_{i_{k}}}{\longrightarrow} \mathbf{x}_{\mathbf{j}_{\mathbf{k}+1}}$ can be associated to a vector $A_{i_{k}} \ldots A_{i_{1}} H_{i}$ and, as we will see later, a complete family traduces the linear combinations of elements $A_{i_{k}} \ldots A_{i_{1}} H_{i}, A_{i_{k}^{\prime}} \ldots A_{i_{1}^{\prime}} H_{i^{\prime}}, \ldots$ which, under some conditions, belongs to $\mathcal{R}_{H}$. More precisely, we have for the present example $P \equiv Q_{1} \odot u_{0} \equiv P_{1} \odot u_{0}$ where $P_{1}$ is a $\mathbf{W}$ - $\boldsymbol{\Gamma}_{\text {int }}$ path. To constitute a complete family associated to $P$, we must add to element $Q_{1} \odot u_{0}$ another one of the form $P_{1}^{\prime} \odot u_{0}$ such that $\alpha\left(P_{1}^{\prime}\right)=\alpha\left(P_{1}\right)$ and $\left\{P_{1}, P_{1}^{\prime}\right\}$ constitutes a $\mathbf{W}-\boldsymbol{\Gamma}_{\mathbf{1}}$ linking of size $t=1$. Since the unique path which attains $\mathbf{x}_{\mathbf{2}}$ is $\mathbf{w}_{\mathbf{2}} \stackrel{u_{0}}{\longrightarrow} \mathbf{x}_{\mathbf{2}} \equiv Q_{2}$, we have that $P_{1}^{\prime} \equiv Q_{2}$. Hence, we call the set $\left\{Q_{1} \odot u_{0}, Q_{2} \odot u_{0}\right\}$ a complete $\mathbf{W}-\boldsymbol{\Gamma}_{\text {int }}$ family. Similarly, $\left\{Q_{1} \odot u_{1}, Q_{2} \odot u_{1}\right\}$ is a complete $\mathbf{W}-\boldsymbol{\Gamma}_{\text {int }}$ family. Naturally, the complete family associated to $P$ includes $P^{\prime}$ and vice-versa.

Now, we give the rigorous definition of a complete family:

- Consider vertex subsets $\mathbf{W}_{\mathbf{0}} \subseteq \mathbf{W} \cup \mathbf{F}$ and $\boldsymbol{\Gamma}_{\text {int }} \subseteq \mathbf{X}_{\mathbf{C}}$ and let $S=\left\{Q_{1} \odot \sigma_{1}, Q_{2} \odot \sigma_{2}, Q_{3} \odot \sigma_{3} \ldots, Q_{k} \odot \sigma_{k}\right\}$, where $\sigma_{1}, \sigma_{2}, \ldots, \sigma_{k}$ belong to $\wp(u)$ and $Q_{1}, \ldots, Q_{k}$ are prime $\mathbf{W}_{\mathbf{0}}-\Gamma_{\text {int }}$ paths which satisfy

- $\forall i \neq j, \alpha\left(Q_{i}\right)=\alpha\left(Q_{j}\right)$ or $Q_{i}$ and $Q_{j}$ are $A$-disjoint.

$S$ is said a complete $\mathbf{W}_{\mathbf{0}}-\boldsymbol{\Gamma}_{\text {int }}$ family when $\forall \ell=1, \ldots, k$, if $Q_{\ell} \odot \sigma_{\ell} \equiv P_{1} \odot \sigma_{1}^{\prime} \equiv P_{2} \odot \sigma_{2}^{\prime} \equiv \ldots \equiv P_{s} \odot \sigma_{s}^{\prime}$, where for $i=$ $1, \ldots, s, P_{i}$ is $\mathbf{W}_{\mathbf{0}}-\boldsymbol{\Gamma}_{\text {int }}$ paths and $\sigma_{i}^{\prime} \neq 1$, then there exist $S_{0}=\left\{Q_{i_{1}} \odot \sigma_{k_{1}} \cdot \sigma_{j_{1}}^{\prime}, Q_{i_{2}} \odot \sigma_{k_{2}} \cdot \sigma_{j_{2}}^{\prime}, \ldots, Q_{i_{t}} \odot \sigma_{k_{t}} \cdot \sigma_{j_{t}}^{\prime}\right\} \subseteq$ $S \backslash\left\{Q_{\ell} \odot \sigma_{\ell}\right\}$, with $\left\{\sigma_{j_{1}}^{\prime}, \sigma_{j_{2}}^{\prime}, \ldots, \sigma_{j_{t}}^{\prime}\right\} \subseteq\left\{\sigma_{1}^{\prime}, \sigma_{2}^{\prime}, \ldots, \sigma_{s}^{\prime}\right\}$, and paths $P_{1}^{\prime} \equiv Q_{i_{1}} \odot \sigma_{k_{1}}, P_{2}^{\prime} \equiv Q_{i_{2}} \odot \sigma_{k_{2}}, \ldots$, $P_{t}^{\prime} \equiv Q_{i_{t}} \odot \sigma_{k_{t}}$, such that

Cond3. $\left\{P_{1}^{\prime}, P_{2}^{\prime}, \ldots, P_{t}^{\prime}\right\}$ constitutes a $\mathbf{W}_{\mathbf{0}^{-}}\left\{\alpha\left(P_{1}\right), \ldots, \alpha\left(P_{s}\right)\right\} A$-disjoint linking of size $t$.

Cond4. the size of the maximal $\mathbf{W}_{0^{-}}\left\{\alpha\left(P_{1}\right), \ldots, \alpha\left(P_{s}\right)\right\} \quad A$-disjoint linking included in $\left\{P_{1}, P_{2}, \ldots, P_{s}, P_{1}^{\prime}, P_{2}^{\prime}, \ldots, P_{t}^{\prime}\right\}$ is equal to $t$.

By definition, empty set $\emptyset$ is a complete family. An illustration of the notion of complete family is given in Example 4.6.

- Consider vertex subsets $\mathbf{W}_{\mathbf{0}} \subseteq \mathbf{W} \cup \mathbf{F}$ and $\boldsymbol{\Gamma}_{\text {int }} \subseteq \mathbf{X}_{\mathbf{C}} . S$ is a minimal complete $\mathbf{W}_{\mathbf{0}}-\boldsymbol{\Gamma}_{\text {int }}$ family if it does not exist a nonempty complete $\mathbf{W}_{\mathbf{0}}-\boldsymbol{\Gamma}_{\text {int }}$ family $S^{\prime} \subset S$.

To compute $g_{-} \operatorname{dim}\left(C \mathcal{R}_{H}\right)$ using this notion of complete family, we need a last definition:

Definition 4.2 Let us denote by $\boldsymbol{\aleph}\left(\mathbf{W}, \boldsymbol{\Gamma}_{\mathbf{1}}, \boldsymbol{\Delta}_{\mathbf{1}}\right)$ the set of all $A$-disjoint $\mathbf{W}-\boldsymbol{\Gamma}_{\mathbf{1}}$ linkings included in $\boldsymbol{\Delta}_{\mathbf{1}}$. For each $\mathbf{W}-\boldsymbol{\Gamma}_{\mathbf{1}}$ path $P$ included in $\boldsymbol{\Delta}_{\mathbf{1}}$, we can associate a minimal complete $\mathbf{W}-\boldsymbol{\Gamma}_{\text {int }}$ family $S(P)$ such that

- $S(P)=\emptyset$ when $P$ is not a strict multiple of any prime $\mathbf{W}-\boldsymbol{\Gamma}_{\text {int }}$ path.

- $\forall Q_{i}$ prime $\mathbf{W}-\boldsymbol{\Gamma}_{\text {int }}$ path and $\forall \sigma_{i} \in \wp(u)$, if $P \equiv Q_{i} \odot \sigma_{i}$, then either $Q_{i} \odot \sigma_{i} \in S(P)$ or $\exists Q_{i}^{\prime} \odot \sigma_{i} \in S(P)$ such that $\alpha\left(Q_{i}\right) \neq \alpha\left(Q_{i}^{\prime}\right)$ and $Q_{i} \equiv Q_{i}^{\prime}$;

- $\forall Q_{i} \odot \sigma_{i} \in S(P)$, length $\left(Q_{i}\right)+$ length $\left(\sigma_{i}\right) \leq \operatorname{card}\left(\boldsymbol{\Delta}_{\mathbf{1}}\right)+\operatorname{card}\left(\boldsymbol{\Delta}_{\mathbf{1}}\right)$. 
- For each $\gamma \subseteq \boldsymbol{\Gamma}_{\mathbf{1}}$, we define the number $\rho_{A}\left(\mathbf{W}, \gamma, \Delta_{\mathbf{1}}, \boldsymbol{\Gamma}_{\mathbf{1}}\right) \stackrel{\text { def }}{=} \max _{L_{0} \in \mathbf{N}\left(\mathbf{W}, \gamma, \mathbf{\Delta}_{\mathbf{1}}\right)}\left[\operatorname{card}\left(L_{0}\right)\right]$ under the constraints:

Ca: $\forall P_{0} \in L_{0}$, if $P_{0} \equiv Q_{0} \odot \sigma_{0}$ then $Q_{0} \odot \sigma_{0} \notin \bigcup_{P \in L_{0} \backslash\left\{P_{0}\right\}} S(P)$,

Cb: either no element of $S(P)$ is strict multiple of any $\mathbf{W}-\boldsymbol{\Gamma}_{\text {ext }}$ path

or if an element of $S(P)$ is a strict multiple of a $\mathbf{W}-\boldsymbol{\Gamma}_{\text {ext }}$ path $P^{\prime}$, then

$$
\max _{L \in \mathbf{N}\left(\mathbf{W}, \boldsymbol{\Gamma}_{\text {ext }} \backslash\left\{\alpha\left(P^{\prime}\right)\right\}, \mathbf{\Delta}_{\mathbf{1}}\right)} \operatorname{card}(L)=\max _{L^{\prime} \in \mathbf{N}\left(\mathbf{W}, \boldsymbol{\Gamma}_{\text {ext }}, \mathbf{\Delta}_{\mathbf{1}}\right)} \operatorname{card}\left(L^{\prime}\right)-1
$$

where $L$ and $L^{\prime}$ contain only paths $\bar{P}$ which satisfy $S(\bar{P})=\emptyset$.

- Finally, we define $\boldsymbol{\Omega}_{\mathbf{1}} \stackrel{\text { def }}{=}\left\{\gamma \subseteq \boldsymbol{\Gamma}_{\mathbf{1}}, \operatorname{card}(\gamma)=\rho_{A}\left(\mathbf{W}, \gamma, \Delta_{\mathbf{1}}, \boldsymbol{\Gamma}_{\mathbf{1}}\right)=\rho_{A}\left(\mathbf{W}, \boldsymbol{\Gamma}_{\mathbf{1}}, \boldsymbol{\Delta}_{\mathbf{1}}, \boldsymbol{\Gamma}_{\mathbf{1}}\right)\right\}$.

Using the previous definitions and settings, we can state the following lemma:

Lemma 4.3 Consider structured bilinear system $\left(\Sigma_{\Lambda}\right)$ represented by digraph $\mathcal{G}\left(\Sigma_{\Lambda}\right)$. Let us denote by $e_{J}$, $e_{\Gamma}$ and $e_{\Delta}$, the diagonal $n \times n$ matrices such that

$e_{J}(i, i)=\left\{\begin{array}{ll}1 & \text { if } \mathbf{x}_{\mathbf{i}} \in \mathbf{J}_{\mathbf{1}} \\ 0 & \text { otherwise }\end{array} e_{\Gamma}(i, i)=\left\{\begin{array}{ll}1 & \text { if } \mathbf{x}_{\mathbf{i}} \in \boldsymbol{\Gamma}_{\mathbf{1}} \\ 0 & \text { otherwise }\end{array} e_{\Delta}(i, i)=\left\{\begin{array}{ll}1 & \text { if } \mathbf{x}_{\mathbf{i}} \in \mathbf{\Delta}_{\mathbf{1}} \\ 0 & \text { otherwise }\end{array}\right.\right.\right.$.

let $\mathcal{R}_{i}$ denote the $i^{\text {th }}$ term of sequence (3), we have generically:

$\forall i \geq 0$, if $e_{J} \mathcal{R}_{i}=0$, then for almost all realizations of $\left(\Sigma_{\Lambda}\right)$, there exists a matrix $B_{1}$ such that

- $\mathcal{M}\left(e_{\Gamma} B_{1}\right)=\mathbf{\Omega}_{\mathbf{1}}$,

$-e_{J} B_{1}=0$,

- $g \_r a n k\left(B_{1}\right)=g \_r a n k\left(e_{\Gamma} B_{1}\right)=\rho_{A}\left(\mathbf{W}, \boldsymbol{\Gamma}_{\mathbf{1}}, \Delta_{\mathbf{1}}, \boldsymbol{\Gamma}_{\mathbf{1}}\right)$,

$-\operatorname{Im}\left(B_{1}\right) \subseteq \mathcal{R}_{H}$,

$-\mathcal{R}_{i} \backslash\left(\mathcal{R}_{i} \cap \operatorname{Im}\left(e_{\Delta}\right)\right) \subseteq \operatorname{Im}\left(B_{1}\right)$.

\section{Proof:}

We first comment the elements of Definition 4.2 as the proof concerns this definition.

Foremost, it is easy to see that if there exist $t A$-disjoint paths noted $P_{1}, P_{2}, \ldots, P_{t}$ between $\left\{\mathbf{w}_{\mathbf{j}_{1}}, \mathbf{w}_{\mathbf{j}_{2}}, \ldots, \mathbf{w}_{\mathbf{j}_{\mathbf{t}}}\right\}$ and $\left\{\mathbf{x}_{\mathbf{i}_{1}}, \mathbf{x}_{\mathbf{i}_{2}}, \ldots, \mathbf{x}_{\mathbf{i}_{\mathbf{t}}}\right\}$ with $\sigma\left(P_{k}\right)=u_{0} \cdot u_{r_{k, 1}} \cdot \ldots \cdot u_{r_{k, l_{k}}}$, for $k=1, \ldots, t$, then $g_{-} \operatorname{dim}\left(\sum_{k=1}^{t} \operatorname{span}\left(A_{r_{k, l_{k}}} \ldots A_{r_{k, 1}} H_{j_{k}}\right)\right)=t$.

Moreover, for each $\left\{\mathbf{w}_{\mathbf{j}}\right\}-\boldsymbol{\Gamma}_{\mathbf{1}}$ path $P$ included in $\Delta_{\mathbf{1}}$ with $\sigma(P)=u_{0} \cdot u_{r_{1}} \cdot \ldots \cdot u_{r_{\ell}}, S(P)=\emptyset$ implies that $A_{r_{\ell}} \ldots A_{r_{1}} H_{j} \in \mathcal{R}_{H}$. Indeed, $S(P)=\emptyset$ is equivalent to say that $P$ is not a strict multiple of a path leading to $\boldsymbol{\Gamma}_{\text {int }}$. This implies $H_{j} \in \operatorname{ker} C, A_{r_{1}} H_{j} \in \operatorname{ker} C, \ldots$, and $A_{r_{\ell-1}} \ldots A_{r_{1}} H_{j} \in \operatorname{ker} C$. Therefore, $A_{r_{\ell}} \ldots A_{r_{1}} H_{j} \in \mathcal{R}_{H}$.

In contrary, the same $\left\{\mathbf{w}_{\mathbf{j}}\right\}-\boldsymbol{\Gamma}_{\mathbf{1}}$ path $P$ included in $\boldsymbol{\Delta}_{\mathbf{1}}$ is such that $P \equiv Q_{1} \odot \sigma_{1}$, where $Q_{1}$ is a prime $\mathbf{W}-\boldsymbol{\Gamma}_{\text {int }}$ path implies that $A_{r_{k}} \ldots A_{r_{1}} H_{j} \notin \operatorname{ker} C$, where $\sigma(P)=u_{0} \cdot u_{r_{1}} \cdot \ldots \cdot u_{r_{k}} \cdot u_{r_{k+1}} \cdot \ldots \cdot u_{r_{\ell}}$ and $\sigma_{1}=u_{r_{k+1}} \cdot \ldots \cdot u_{r_{\ell}}$.

Consider now an $\mathbf{W}-\boldsymbol{\Gamma}_{\mathbf{1}}$ path $P$ included in $\Delta_{\mathbf{1}}$ and its associated complete $\mathbf{W}-\boldsymbol{\Gamma}_{\text {int }}$ family $S(P)=$ $\left\{Q_{1} \odot \sigma_{1}, Q_{2} \odot \sigma_{2}, \ldots, Q_{k} \odot \sigma_{k}\right\}$ satisfying condition $\mathbf{C b}$. We show hereafter that $\left(\sum_{s=1}^{k} \operatorname{span}\left(A_{r_{s, \ell_{s}}} \ldots A_{r_{i, 1}} H_{j_{s}}\right)\right) \cap$ $\mathcal{R}_{H} \neq 0$, where for $s=1, \ldots, k, \sigma\left(Q_{s}\right) \cdot \sigma_{s}=u_{0} \cdot u_{r_{s, 1}} \cdot \ldots \cdot u_{r_{s, \ell_{s}}}$ and $\mathbf{w}_{\mathbf{j}_{\mathrm{s}}}$ is the begin vertex of $Q_{s}$ :

At first, due to constraint length $\left(Q_{s}\right)+\operatorname{length}\left(\sigma_{s}\right) \leq \operatorname{card}\left(\boldsymbol{\Delta}_{\mathbf{1}}\right)+\operatorname{card}\left(\boldsymbol{\Gamma}_{\mathbf{1}}\right), S(P)$ has a finite number of elements. Moreover, let us consider in $S(P)$ an element noted $Q_{1} \odot \sigma_{1}$, where $Q_{1}$ is a prime $\mathbf{W}-\boldsymbol{\Gamma}_{\text {int }}$ path and such that 
length $\left(\sigma_{1}\right)$ is maximal in $S(P)$. Even if this element is not unique, it exists since $S(P)$ has a finite number of elements. We denote this element $Q_{1} \odot \sigma_{1}$ only for the sake of simplicity and in order to avoid an additional index. To do so, an eventual reordering of $S(P)$ may be necessary.

Let us take the following notations, $\sigma\left(Q_{1}\right)=u_{0} \cdot u_{r_{1,1}} \cdot \ldots \cdot u_{r_{1, \ell_{1}}}, \sigma_{1}=u_{r_{1, \ell_{1}+1}} \ldots \cdot u_{r_{1, k_{1}}}$ and $v_{1}=A_{r_{1, \ell_{1}}} \ldots A_{r_{1,1}} H_{j_{1}}$, where $\mathbf{w}_{\mathbf{j}_{\mathbf{1}}}$ is the begin vertex of $Q_{1}$. Obviously, $v_{1} \cap \operatorname{ker} C=0$ and $v_{1} \in \mathcal{R}_{H}$.

As $S(P)$ is complete, there exist some elements denoted, for the sake of simplicity, $Q_{2} \odot \sigma_{2}, Q_{3} \odot \sigma_{3}, \ldots, Q_{t+1} \odot \sigma_{t+1}$ which satisfy conditions Cond3 and Cond4. Let us note for $s=2, \ldots, t+1, \sigma\left(Q_{s}\right)=u_{0} \cdot u_{r_{s, 1}} \cdot \ldots \cdot u_{r_{s, s}}$, $\sigma_{s}=u_{r_{s, \ell_{s}+1}} \cdot \ldots \cdot u_{r_{s, k_{s}}}$ and $v_{s}=A_{r_{s, \ell_{s}}} A_{r_{s, \ell_{s}-1}} \ldots A_{r_{s, 1}} H_{j_{s}}$, where $\mathbf{w}_{\mathbf{j}_{\mathrm{s}}}$ is the begin vertex of $Q_{s}$. Note that as they are prime $\mathbf{W}-\boldsymbol{\Gamma}_{\text {int }}$ paths, $Q_{s}$, for $s=1, \ldots, t+1$ can not be strict multiple of $\mathbf{W}-\boldsymbol{\Gamma}_{\text {ext }}$ paths.

Moreover, we have for elements $Q_{s}, s=1, \ldots, t+1$, only two possible cases:

- case 1: $\forall s=1, \ldots, t+1, Q_{s}$ is not a multiple of any $\mathbf{W}-\boldsymbol{\Gamma}_{\text {ext }}$ path. In this case, all vectors $A_{r_{1, \ell}} v_{s}, s=1, \ldots, t+1$, associated to $Q_{s} \odot u_{r_{1, \ell_{1}+1}}$ are such that $e_{J} v_{s}=0$.

Moreover, since, $\forall s=2, \ldots, t+1$, length $\left(\sigma_{s}\right) \leq$ length $\left(\sigma_{1}\right)$ and due to conditions Cond3 and Cond4, we have that $v_{s} \in \mathcal{R}_{H}$ and $\sigma_{s}=\sigma_{1}$. Furthermore, these conditions Cond3 and Cond4 imply also that $g_{-} \operatorname{dim}\left(\sum_{s=1}^{t+1} \operatorname{span}\left(v_{s}\right)\right)=t+1, g_{-} \operatorname{dim}\left(C \sum_{s=1}^{t+1} \operatorname{span}\left(v_{s}\right)\right)=t$ and $g_{-} \operatorname{dim}\left(C \sum_{s=2}^{t+1} \operatorname{span}\left(v_{s}\right)\right)=t$. Therefore, $\sum_{s=1}^{t+1} \operatorname{span}\left(v_{s}\right) \cap \operatorname{ker} C \neq 0$. Since $\sum_{s=1}^{t+1} \operatorname{span}\left(v_{s}\right) \subseteq \mathcal{R}_{H}$, there exists $v_{1}^{\prime} \in \sum_{s=1}^{t+1} \operatorname{span}\left(v_{s}\right)$ such that $A_{r_{1, \ell_{1}+1}} v_{1}^{\prime} \in \mathcal{R}_{H}$ and $e_{J} A_{r_{1, \ell_{1}+1}} v_{1}^{\prime}=0$.

- case 2: $\exists s$ such that $Q_{s}$ is a multiple of a $\mathbf{W}-\boldsymbol{\Gamma}_{\mathbf{e x t}}$ path $P^{\prime}$. Due to constraint $\mathbf{C b}, e_{i_{1}} \in \mathcal{R}_{H}$, where $\mathbf{x}_{\mathbf{i}_{1}}=\alpha\left(P^{\prime}\right)$ and $e_{i_{1}}$ is the $i_{1}^{t h}$ Euclidean vector. Obviously, $e_{i_{1}} \in \operatorname{Im}\left(e_{\Gamma}\right)$.

For all the $Q_{s} \odot \sigma_{s}$ which are multiple of $\mathbf{W}-\Gamma_{\text {ext }}$ paths, we join to $\left\{Q_{1} \odot u_{r_{1, \ell_{1}+1}}, Q_{2} \odot u_{r_{1, \ell_{1}+1}}, \ldots, Q_{t} \odot u_{r_{1, \ell_{1}+1}}\right\}$, the path subset $\left\{\bar{P}_{1} u_{r_{1, \ell_{1}+1}}, \ldots, \bar{P}_{t^{\prime}} u_{r_{1, \ell_{1}+1}}\right\}$ where $L=\left\{\bar{P}_{1}, \ldots, \bar{P}_{t^{\prime}}\right\}$ is the linking described in $\mathbf{C b}$. Let us denote by $\bar{v}_{s^{\prime}}$ the vector associated to each $\bar{P}_{s^{\prime}}, s^{\prime}=1, \ldots, t^{\prime}$. We can deduce from $\mathbf{C b}$, conditions $\mathbf{C o n d} \mathbf{3}$ and $\mathbf{C o n d} 4$ that, $\bar{v}_{s^{\prime}} \in \mathcal{R}_{H} \cap \operatorname{Im}\left(e_{\Gamma}\right), g_{-} \operatorname{dim}\left(\sum_{s=1}^{t+1} \operatorname{span}\left(v_{s}\right)+\sum_{s^{\prime}=1}^{t^{\prime}} \operatorname{span}\left(\bar{v}_{s^{\prime}}\right)\right)=t^{\prime}+t+1, g_{-} \operatorname{dim} e_{J}\left(\sum_{s=1}^{t+1} \operatorname{span}\left(v_{s}\right)+\sum_{s^{\prime}=1}^{t^{\prime}} \operatorname{span}\left(\bar{v}_{s^{\prime}}\right)\right)=0$ and $g_{-} \operatorname{dim}\left(\begin{array}{c}c \\ e_{\Gamma}\end{array}\right)\left(\sum_{s=1}^{t+1} \operatorname{span}\left(v_{s}\right)+\sum_{s^{\prime}=1}^{t^{\prime}} \operatorname{span}\left(\bar{v}_{s^{\prime}}\right)\right)=t+t^{\prime}$. Using similar arguments than previously, we have that $\left(\sum_{s=1}^{t+1} \operatorname{span}\left(v_{s}\right)+\sum_{s^{\prime}=1}^{t^{\prime}} \operatorname{span}\left(\bar{v}_{s^{\prime}}\right)\right) \cap\left(\operatorname{ker} C \cap \operatorname{Im}\left(e_{\Delta}\right)\right) \neq 0$.

Since $\sum_{s=1}^{t+1} \operatorname{span}\left(v_{s}\right)+\sum_{s^{\prime}=1}^{t^{\prime}} \operatorname{span}\left(\bar{v}_{s^{\prime}}\right) \subseteq \mathcal{R}_{H}$, there exists $v_{1}^{\prime} \in \sum_{s=1}^{t+1} \operatorname{span}\left(v_{s}\right)+\sum_{s^{\prime}=1}^{t^{\prime}} \operatorname{span}\left(\bar{v}_{s}^{\prime}\right)$ such that $A_{r_{1, \ell_{1}+1}} v_{1}^{\prime} \in \mathcal{R}_{H}$ and $v_{1}^{\prime} \in \operatorname{Im}\left(e_{\Delta}\right)$ which implies that $e_{J} A_{r_{1, \ell_{1}+1}} v_{1}^{\prime}=0$.

To summarize, in the two previous cases, we have that we can extract from the vectors associated to $\left\{Q_{1} \odot u_{r_{1, \ell_{1}+1}}, Q_{2} \odot u_{r_{1, \ell_{1}+1}}, \ldots, Q_{t} \odot u_{r_{1, \ell_{1}+1}}\right\}$ a vector $v_{1}^{\prime}$ such that $A_{r_{1, \ell_{1}+1}} v_{1}^{\prime} \in \mathcal{R}_{H}$ and $e_{J} A_{r_{1, \ell_{1}+1}} v_{1}^{\prime}=0$.

In fact, in $S(P)$, the presence of elements $Q_{s} \odot \sigma_{s}, s=1, \ldots, t+1$, satisfying $\mathbf{C a}$ and $\mathbf{C b}$, is equivalent to the presence of $Q_{1}^{\prime} \odot \sigma_{1}^{\prime}$, where $\sigma_{1}^{\prime}=u_{r_{1, \ell_{1}+2}} \cdot \ldots \cdot u_{r_{1, k_{1}}}$ is "shorter" than $\sigma_{1}$ and $Q_{1}^{\prime}$ could be represented by 
$\left\{Q_{1} \odot u_{r_{1, \ell_{1}+1}}, Q_{2} \odot u_{r_{1, \ell_{1}+1}}, \ldots, Q_{t} \odot u_{r_{1, \ell_{1}+1}}\right\}$ which traduces the existence of a nonzero vector $v_{1}^{\prime} \in \sum_{s=1}^{t+1} \operatorname{span}\left(v_{s}\right) \cap \mathcal{R}_{H}$ satisfying $e_{J} v_{1}^{\prime}=0$ since $v_{1}^{\prime} \in \operatorname{Im}\left(e_{\Delta_{1}}\right)$.

We can repeat this reasoning, until obtaining a final element $Q^{\left(k^{\prime}\right)} \sigma_{k}^{\prime \prime}$, where $\sigma_{1}^{\left(k^{\prime}\right)}=1$ and $Q^{\left(k^{\prime}\right)}$ traduces the fact that there exists a nonzero vector $v_{k}^{\prime} \in\left(\sum_{s=1}^{k} \operatorname{span}\left(A_{r_{s, \ell_{s}}} \ldots A_{r_{s, 2}} A_{r_{s, 1}} H_{j_{s}}\right)\right) \cap \mathcal{R}_{H}$ with $e_{J} v_{k}^{\prime}=0$.

Therefore, for each path $P$ such that either $S(P)=\emptyset$ or $S(P)$ verifies constraint $\mathbf{C b}$, we can associate a vector $v^{\prime} \in \mathcal{R}_{H}$ satisfying $e_{J} v^{\prime}=0$. In this case, we have obviously, $\mathcal{M}\left(e_{\Gamma} v^{\prime}\right)=\left\{\left\{\alpha\left(P_{i}\right)\right\}, P_{i} \equiv Q_{i} \odot \sigma_{i} \in S(P)\right.$ and $\left.\alpha\left(P_{i}\right) \in \boldsymbol{\Gamma}_{\mathbf{1}}\right\}$. Since $P$ is an $\mathbf{W}-\Gamma$ path and as $S(P)$ is minimal, we have necessarily $e_{\Gamma} v^{\prime} \neq 0$.

Consider now any set $\mathcal{S}=\bigcup_{P \in L_{0}}\{S(P)\}$ of complete families $S(P)$. If $S(P), \forall P \in L_{0}$, is minimal and satisfies constraint $\mathbf{C a}$ and since all the paths $P \in L_{0}$ are $A$-disjoint, then all vectors $v^{\prime}$ associated to each subset $S(P)$ are generically linearly independent. Moreover, as we have shown it above, if $S(P), \forall P \in L_{0}$, is minimal and satisfies constraint $\mathbf{C b}$, then all vectors $v^{\prime}$ associated to each subset $S(P)$ are generically such that $v^{\prime} \in \mathcal{R}_{H}, e_{J} v^{\prime}=0$ and $e_{\Gamma} v^{\prime} \neq 0$.

Hence, if we consider matrix $B_{1}$ defined by the concatenation of all these independent vectors $v^{\prime}$, then we have obviously that $\mathcal{M}\left(e_{\Gamma} B_{1}\right)=\mathbf{\Omega}_{\mathbf{1}}, g_{-} \operatorname{rank}\left(B_{1}\right)$ is equal to the number of vectors associated to paths $P \in L_{0}$ and since all these vectors are such that $e_{\Gamma} v^{\prime} \neq 0$ so $g_{-} \operatorname{rank}\left(e_{\Gamma} B_{1}\right)=g_{-} \operatorname{rank}\left(B_{1}\right)=\rho_{A}\left(\mathbf{W}, \boldsymbol{\Gamma}_{\mathbf{1}}, \Delta_{\mathbf{1}}, \boldsymbol{\Gamma}_{\mathbf{1}}\right)$. Moreover, $\operatorname{Im}\left(B_{1}\right) \subseteq \mathcal{R}_{H}$ and $e_{J} B_{1}=0$ and so the first four points of Lemma 4.3 are proved.

To prove the last point of Lemma 4.3, we use a proof by contradiction in assuming that for some $i \geq 0, e_{J} \mathcal{R}_{i}=0$ and $\mathcal{R}_{i} \backslash\left(\mathcal{R}_{i} \cap \operatorname{Im}\left(e_{\Delta}\right)\right) \nsubseteq \operatorname{Im}\left(B_{1}\right)$. This means obviously that $\exists v_{0} \in \mathcal{R}_{i}$ such that $e_{\Gamma} v_{0} \notin \operatorname{Im}\left(e_{\Gamma} B_{1}\right)$. Let $B_{1}^{\prime}$ be the matrix obtained by the concatenation of $B_{1}$ and $v_{0}$ i.e. $B_{1}^{\prime}=\left(B_{1} \mid v_{0}\right)$. Obviously, there exists a subset $\gamma^{\prime}=\gamma \cup\left\{\mathbf{x}_{\mathbf{i}_{0}}\right\} \in \mathcal{M}\left(e_{\Gamma} B_{1}^{\prime}\right)$, where $\gamma \in \mathcal{M}\left(e_{\Gamma} B_{1}\right), \mathbf{x}_{\mathbf{i}_{0}} \in \boldsymbol{\Gamma}_{\mathbf{1}}$ and $\left\{\mathbf{x}_{\mathbf{i}_{0}}\right\} \in \mathcal{M}\left(v_{0}\right)$. Recall that $\gamma \in \mathcal{M}\left(e_{\Gamma} B_{1}\right)$ is equivalent to say that $\rho_{A}\left(\mathbf{W}, \gamma, \Delta_{\mathbf{1}}, \boldsymbol{\Gamma}_{\mathbf{1}}\right)=\rho_{A}\left(\mathbf{W}, \boldsymbol{\Gamma}_{\mathbf{1}}, \Delta_{\mathbf{1}}, \boldsymbol{\Gamma}_{\mathbf{1}}\right)$.

Let $P_{0,1}, P_{0,2}, \ldots, P_{0, k_{0}}$ all the $\mathbf{W}$ - $\left\{\mathbf{x}_{\mathbf{i}_{0}}\right\}$ paths included in $\boldsymbol{\Delta}_{\mathbf{1}}$ and such that $\operatorname{length}\left(P_{0, j}\right) \leq i, \forall j=1, \ldots, k_{0}$. Obviously, since $v_{0}^{\prime} \in \mathcal{R}_{i}$ and $e_{J} \mathcal{R}_{i}=0$, there exists at least a path satisfying conditions above.

Let $\mathcal{S}_{1}, \mathcal{S}_{2}, \ldots, \mathcal{S}_{k}$ be all the sets of minimal complete families $\mathcal{S}_{\ell}=\bigcup_{P \in L_{0}}\{S(P)\}$, satisfying constraints $\mathbf{C a}$ and $\mathbf{C b}$ with $\alpha\left(L_{0}\right)=\gamma$. In fact, since there can exist many $A$-disjoint linkings $L_{0}$ with $\alpha\left(L_{0}\right)=\gamma$ and as for one path $P$ we can associate different $S(P)$, there can exist many sets $\mathcal{S}_{\ell}$.

Only three cases have to be distinguished:

- case 1: there exists a path $P_{0, j}$ associated to a minimal complete family $S\left(P_{0, j}\right)=\emptyset$ and such that $L_{0}^{\prime}=L_{0} \cup\left\{P_{0, j}\right\}$ is $A$-disjoint.

- case 2: there exists a path $P_{0, j}$ associated to a minimal complete family $S\left(P_{0, j}\right) \neq \emptyset$ (i.e. $\mathbf{x}_{\mathbf{i}_{0}} \in \boldsymbol{\Gamma}_{\text {ext }}$ ) satisfying constraint $\mathbf{C b}$, a set of complete family $\mathcal{S}_{\ell}$ such that, $\forall S(P) \in \mathcal{S}_{\ell}$ and $\forall Q_{i} \odot \sigma_{i} \in S(P), P_{0, j} \not \equiv Q_{i} \odot \sigma_{i}$ and $L_{0}^{\prime}=L_{0} \cup\left\{P_{0, j}\right\}$ is $A$-disjoint.

In the two first cases, $\mathcal{S}^{\prime}=\bigcup_{P \in L_{0}^{\prime} \cup\left\{P_{0, j}\right\}} S(P)$ satisfies constraints of linear independence Ca. Thus, linking $L_{0}^{\prime}$ satisfies constraints of Definition 4.2 and $\rho_{A}\left(\mathbf{W}, \boldsymbol{\Gamma}_{\mathbf{1}}, \boldsymbol{\Delta}_{\mathbf{1}}, \boldsymbol{\Gamma}_{\mathbf{1}}\right)=\rho_{A}\left(\mathbf{W}, \gamma, \boldsymbol{\Delta}_{\mathbf{1}}, \boldsymbol{\Gamma}_{\mathbf{1}}\right)<\operatorname{card}\left(L_{0}^{\prime}\right)=\rho_{A}\left(\mathbf{W}, \gamma^{\prime}, \boldsymbol{\Delta}_{\mathbf{1}}, \boldsymbol{\Gamma}_{\mathbf{1}}\right)$, with 
$\gamma^{\prime} \subseteq \boldsymbol{\Gamma}_{\mathbf{1}}$. This is in contradiction with Definition 4.2. Indeed, $\rho_{A}\left(\mathbf{W}, \boldsymbol{\Gamma}_{\mathbf{1}}, \boldsymbol{\Delta}_{\mathbf{1}}, \boldsymbol{\Gamma}_{\mathbf{1}}\right)$ is the cardinality of the biggest $A$-disjoint linking which satisfies all constraints of Definition 4.2. Therefore, for these two first cases, we cannot have $e_{\Gamma} v_{0} \notin \operatorname{Im}\left(e_{\Gamma} B_{1}\right)$ and so $\mathcal{R}_{i} \backslash\left(\mathcal{R}_{i} \cap \operatorname{Im}\left(e_{\Delta}\right)\right) \subseteq \operatorname{Im}\left(B_{1}\right)$.

- case 3: $\forall j, \forall \ell, S\left(P_{0, j}\right) \neq \emptyset$ and $\exists S(P) \in \mathcal{S}_{\ell}$ such that $\exists Q_{k} \odot \sigma_{k} \in S(P), P_{0, j} \equiv Q_{k} \odot \sigma_{k}$. This implies, according to the fact that $S(P)$ satisfies constraint $\mathbf{C a}$ and due to the minimality of $S(P) \in \mathcal{S}_{\ell}$, that vector $v_{0, j}$ associated to $P_{0, j}$ belongs generically to $\operatorname{Im}\left(B_{1}\right)$. Indeed, in this case $S(P)$ can also be associated to $P_{0, j}$. This is true for all paths $P_{0, j}, j=1, \ldots k_{0}$ i.e. all $\mathbf{W}$ - $\left\{\mathbf{x}_{\mathbf{i}_{0}}\right\}$ paths included in $\Delta_{\mathbf{1}}$. Therefore, $\forall v \in \mathcal{R}_{i}$ such that $\left\{\mathbf{x}_{\mathbf{i}_{0}}\right\} \in \mathcal{M}(v)$, we have that $v \in \operatorname{Im}\left(B_{1}\right)$. In particular $v_{0} \in \operatorname{Im}\left(B_{1}\right)$.

Therefore, all the possible three cases lead to $\mathcal{R}_{i} \backslash\left(\mathcal{R}_{i} \cap \operatorname{Im}\left(e_{\Delta}\right)\right) \subseteq \operatorname{Im}\left(B_{1}\right)$ and the lemma follows.

Roughly speaking, the object of Lemma 4.3 is to establish that the obtained set $\boldsymbol{\Omega}_{\mathbf{1}}$ can be generically associated to a structured matrix denoted $B_{1}$ which summarizes the influence of the propagation of the perturbation $w$ until it reaches the state components in $\mathbf{J}_{\mathbf{1}}$. Defining $B_{1}$ on the basis of $\boldsymbol{\Omega}_{\mathbf{1}}$ such that $\mathcal{M}\left(e_{\Gamma} B_{1}\right)=\mathbf{\Omega}_{\mathbf{1}}, e_{J} B_{1}=0$ and $e_{\Gamma} B_{1, j} \neq 0$ $\forall j$, where $B_{1, j}$ is the $j^{\text {th }}$ column of $B_{1}$, implies necessarily that $g_{-} \operatorname{rank}\left(B_{1}\right)=g_{-} \operatorname{rank}\left(e_{\Gamma} B_{1}\right)=\rho_{A}\left(\mathbf{W}, \boldsymbol{\Gamma}_{\mathbf{1}}, \Delta_{\mathbf{1}}, \boldsymbol{\Gamma}_{\mathbf{1}}\right)$. Moreover, $\operatorname{Im}\left(B_{1}\right) \subseteq \mathcal{R}_{H}$, implies that structured matrix $B_{1}$ does not contain any new directions w.r.t. $\mathcal{R}_{H}$. Finally, as long as $e_{J} \mathcal{R}_{i}=0, \mathcal{R}_{i} \backslash\left(\mathcal{R}_{i} \cap \operatorname{Im}\left(e_{\Delta}\right)\right) \subseteq \operatorname{Im}\left(B_{1}\right)$ implies that as long as $e_{J} \mathcal{R}_{i}=0, B_{1}$ represents all the influence of the perturbation $w$ on $\boldsymbol{\Gamma}_{\mathbf{1}}$ and so on the outputs since $\mathbf{X}_{\mathbf{c}} \subseteq \boldsymbol{\Gamma}_{\mathbf{1}} \cup \mathbf{J}_{\mathbf{1}}$. Thus, as long as $e_{J} \mathcal{R}_{i}=0$, we obtain the same generic dimensions of subspaces $e_{\Gamma} \mathcal{R}_{i}$ and $C \mathcal{R}_{i}$ if we replace virtually matrix $H$ by matrix $B_{1}$.

We can deduce from the previous lemma:

Lemma 4.4 Consider structured bilinear system $\left(\Sigma_{\Lambda}\right)$ represented by digraph $\mathcal{G}\left(\Sigma_{\Lambda}\right)$. We have generically:

$\forall i \geq 0$, if $e_{J} \mathcal{R}_{i}=0$, then there exist generically a subspace $U_{1} \subseteq \operatorname{Im}\left(e_{\Delta}\right) \subseteq \operatorname{ker} C$ and a matrix $B_{1}$ satisfying conditions of Lemma 4.3 (i.e. $\mathcal{M}\left(e_{\Gamma} B_{1}\right)=\mathbf{\Omega}_{\mathbf{1}}, e_{J} B_{1}=0$, g_rank $\left.\left(B_{1}\right)=\rho_{A}\left(\mathbf{W}, \boldsymbol{\Gamma}_{\mathbf{1}}, \Delta_{\mathbf{1}}, \boldsymbol{\Gamma}_{\mathbf{1}}\right), \operatorname{Im}\left(B_{1}\right) \subseteq \mathcal{R}_{H}\right)$ and such - $\sum_{j=0}^{\text {that }} A_{j} U_{1} \subseteq U_{1}+\operatorname{Im}\left(B_{1}\right)$,

$-\mathcal{R}_{i} \subseteq U_{1}+\operatorname{Im}\left(B_{1}\right)$

\section{Proof:}

$e_{J} \mathcal{R}_{i}=0$ implies that $\mathcal{R}_{i}=\left(\mathcal{R}_{i} \cap \operatorname{Im}\left(e_{\Delta}\right)\right)+\mathcal{R}_{\Gamma}$ where, from Lemma 4.3, there exists a matrix $B_{1}$ satisfying conditions of Lemma 4.3 such that $\mathcal{R}_{\Gamma} \subseteq \operatorname{Im}\left(B_{1}\right)$. Let us denote $\mathcal{R}_{i} \cap \operatorname{Im}\left(e_{\Delta}\right)$ by $\mathcal{D}_{0}$ and let us define $\mathcal{D}_{i+1}=$ $\mathcal{D}_{i}+\sum_{j=0}^{m} A_{j}\left(\mathcal{D}_{i} \cap \operatorname{Im}\left(e_{\Delta}\right)\right)$. The non-decreasing sequence $\mathcal{D}_{i}$ has a unique limit noted $\mathcal{D}_{*}$.

By construction $\forall i \geq 0, e_{J} \mathcal{D}_{i}=0$ and using similar arguments to the ones of Lemma 4.3, $\mathcal{D}_{i} \backslash\left(\mathcal{D}_{i} \cap \operatorname{Im}\left(e_{\Delta}\right)\right) \subseteq$ $\operatorname{Im}\left(B_{1}\right)$. Indeed, sequence $\mathcal{D}_{i}$ is equivalent to sequence $\mathcal{R}_{i}$ in the specific case where all the $A_{i}$-edges, $i=0, \ldots, m$, starting from the elements of $\boldsymbol{\Gamma}_{\mathbf{1}}$, are removed. As we have proved that $e_{J} \mathcal{R}_{i}=0 \Rightarrow \mathcal{R}_{i} \backslash\left(\mathcal{R}_{i} \cap \operatorname{Im}\left(e_{\Delta}\right)\right) \subseteq \operatorname{Im}\left(B_{1}\right)$ in the general case, then it is also true in the specific case where all the $A_{i}$-edges, $i=0, \ldots, m$, starting from vertex subset $\Gamma_{1}$ are removed and so $e_{J} \mathcal{D}_{i}=0 \Rightarrow \mathcal{D}_{i} \backslash\left(\mathcal{D}_{i} \cap \operatorname{Im}\left(e_{\Delta}\right)\right) \subseteq \operatorname{Im}\left(B_{1}\right)$. Therefore, $\mathcal{D}_{*} \backslash\left(\mathcal{D}_{*} \cap \operatorname{Im}\left(e_{\Delta}\right)\right) \subseteq \operatorname{Im}\left(B_{1}\right)$. Moreover, $\sum_{j=0}^{m} A_{j}\left(\mathcal{D}_{*} \cap \operatorname{Im}\left(e_{\Delta}\right)\right) \subseteq \mathcal{D}_{*}$. So, if we denote $\mathcal{D}_{*} \cap \operatorname{Im}\left(e_{\Delta}\right)$ by $U_{1}$, then we have that $\sum_{j=0}^{m} A_{j} U_{1} \subseteq \mathcal{D}_{*} \subseteq$ $U_{1}+\operatorname{Im}\left(B_{1}\right)$. 
Moreover, since $\mathcal{R}_{i} \cap \operatorname{Im}\left(e_{\Delta}\right)=\mathcal{D}_{0} \subseteq \mathcal{D}_{*}$, and since $\mathcal{R}_{\Gamma} \subseteq \operatorname{Im}\left(B_{1}\right)$, we also have $\mathcal{R}_{i} \subseteq U_{1}+\operatorname{Im}\left(B_{1}\right)$ and the lemma follows.

We can deduce from the last lemma, the following corollary, which allows to characterize $\mathcal{R}_{H}$ :

Corollary 4.5 Consider $\operatorname{SBLS}\left(\Sigma_{\Lambda}\right)$ represented by digraph $\mathcal{G}\left(\Sigma_{\Lambda}\right)$.

St1. for almost all structured matrices $B$ such that $\mathcal{M}\left(e_{\Gamma} B\right)=\mathbf{\Omega}_{1}$, we have that $g_{-} \operatorname{dim}\left(C \mathcal{R}_{H}\right)=g_{-} \operatorname{dim}\left(C \mathcal{R}_{B}\right)$, where $\mathcal{R}_{B_{1}}$ is the minimum invariant $\left(C-\left(A_{0}, \ldots, A_{m}\right)\right)$ subspace including $\operatorname{Im}\left(B_{1}\right)$.

St2. if $\rho_{A}\left(\mathbf{W}, \boldsymbol{\Gamma}_{\mathbf{1}}, \boldsymbol{\Delta}_{\mathbf{1}}, \boldsymbol{\Gamma}_{\mathbf{1}}\right)=\rho(\mathbf{W}, \mathbf{Y})$ then $g_{-} \operatorname{dim}\left(C \mathcal{R}_{H}\right)=\rho_{A}\left(\mathbf{W}, \boldsymbol{\Gamma}_{\mathbf{1}}, \boldsymbol{\Delta}_{\mathbf{1}}, \boldsymbol{\Gamma}_{\mathbf{1}}\right)$.

\section{Proof:}

St1. This statement can be proved using mathematical induction. According to Lemma 4.4 and to the fact that $e_{J} \mathcal{R}_{0}=0$, we have obviously that there exists a structured matrix $B_{1}$ such that $\mathcal{R}_{0} \subseteq U_{1}+\operatorname{Im}\left(B_{1}\right) \subseteq U_{1}+\mathcal{R}_{B_{1}}$, where $\mathcal{R}_{B_{1}}$ is the minimum invariant $\left(C-\left(A_{0}, \ldots, A_{m}\right)\right)$ subspace including $\operatorname{Im}\left(B_{1}\right)$. Assume that $\mathcal{R}_{i_{0}} \subseteq U_{1}+\mathcal{R}_{B_{1}}$. From sequence (3), we have that $\mathcal{R}_{i_{0}+1}=\mathcal{R}_{i_{0}}+\sum_{j=0}^{m} A_{j}\left(\mathcal{R}_{i_{0}} \cap \operatorname{ker} C\right)$. As $U_{1} \subseteq \operatorname{ker} C$ and $\sum_{j=0}^{m} A_{j} U_{1} \subseteq U_{1}+\operatorname{Im}\left(B_{1}\right)$, we can write that $\mathcal{R}_{i_{0}+1} \subseteq U_{1}+\mathcal{R}_{B_{1}}+U_{1}+\operatorname{Im}\left(B_{1}\right)+\sum_{j=0}^{m} A_{j}\left(\mathcal{R}_{B_{1}} \cap \operatorname{ker} C\right) \subseteq U_{1}+\mathcal{R}_{B_{1}}$. Therefore, $\mathcal{R}_{H} \subseteq U_{1}+\mathcal{R}_{B_{1}}$. Furthermore, as $\operatorname{Im}\left(B_{1}\right) \subseteq \mathcal{R}_{H}$, which implies that $\mathcal{R}_{B_{1}} \subseteq \mathcal{R}_{H}$, we have that $\mathcal{R}_{B_{1}} \subseteq \mathcal{R}_{H} \subseteq U_{1}+\mathcal{R}_{B_{1}}$. Thus $C \mathcal{R}_{B_{1}} \subseteq C \mathcal{R}_{H} \subseteq C\left(U_{1}+\mathcal{R}_{B_{1}}\right)$ and as $U_{1} \subseteq \operatorname{ker} C$, we obtain $C \mathcal{R}_{H}=C \mathcal{R}_{B_{1}}$. Then, $g_{-} \operatorname{dim}\left(C \mathcal{R}_{H}\right)=g_{-} \operatorname{dim}\left(C \mathcal{R}_{B_{1}}\right)$. Since this equality is true for at least one structured matrix $B_{1}$, it is also true for almost all structured matrices $B$ such that $\mathcal{M}\left(e_{\Gamma} B\right)=\mathbf{\Omega}_{\mathbf{1}}$.

St2. $\rho_{A}\left(\mathbf{W}, \boldsymbol{\Gamma}_{\mathbf{1}}, \boldsymbol{\Delta}_{\mathbf{1}}, \boldsymbol{\Gamma}_{\mathbf{1}}\right)=\rho(\mathbf{W}, \mathbf{Y})$ is equivalent to the fact that there exists a structured matrix $B_{1}$ satisfying conditions of Lemma 4.4 such that $g_{-} \operatorname{rank}\left(B_{1}\right)=g_{-} \operatorname{rank}\left(e_{\Gamma} B_{1}\right)=\rho_{A}\left(\mathbf{W}, \boldsymbol{\Gamma}_{\mathbf{1}}, \boldsymbol{\Delta}_{\mathbf{1}}, \boldsymbol{\Gamma}_{\mathbf{1}}\right)=\rho(\mathbf{W}, \mathbf{Y})$.

On the one hand, from definition of $\boldsymbol{\Gamma}_{\mathbf{1}}$ and $\boldsymbol{\Delta}_{\mathbf{1}},\left(\boldsymbol{\Gamma}_{\mathbf{1}} \cup \boldsymbol{\Delta}_{\mathbf{1}}\right) \cap \mathbf{X}_{\mathbf{c}}=\boldsymbol{\Gamma}_{\mathbf{1}} \cap \mathbf{X}_{\mathbf{C}}$. Moreover, due to the structure of matrix $C$, we have that $\rho(\mathbf{W}, \mathbf{Y})=\rho\left(\mathbf{W}, \mathbf{X}_{\mathbf{c}} \cap \boldsymbol{\Gamma}_{\mathbf{1}}\right)$. On the other hand, every maximal $\mathbf{W}-\boldsymbol{\Gamma}_{\mathbf{1}} \cap \mathbf{X}_{\mathbf{C}}$ linking can be associated to complete families such that constraint $\mathbf{C a}$ is satisfied. Therefore, for each maximal $\mathbf{W}-\boldsymbol{\Gamma}_{\mathbf{1}} \cap \mathbf{X}_{\mathbf{C}}$ linking $\mathcal{L}$, there exists $\gamma \in \mathbf{\Omega}_{\mathbf{1}}$, such that $\alpha(\mathcal{L}) \subseteq \gamma$. Yet, $\rho(\mathbf{W}, \mathbf{Y}) \leq g_{-} \operatorname{rank}\left(C B_{1}\right)=\max _{\gamma \in \mathbf{\Omega}_{\mathbf{1}}, \gamma \subseteq \mathbf{X}_{\mathbf{C}}} \operatorname{card}(\gamma) \leq \max _{\gamma \in \mathbf{\Omega}_{\mathbf{1}}} \operatorname{card}(\gamma)$. Consequently, we have in general that $\rho(\mathbf{W}, \mathbf{Y}) \leq g_{-} \operatorname{rank}\left(C B_{1}\right) \leq g_{-} \operatorname{rank}\left(B_{1}\right)$.

Thus, if equality of $\mathbf{S t} \mathbf{2}$ is satisfied i.e. $\rho_{A}\left(\mathbf{W}, \boldsymbol{\Gamma}_{\mathbf{1}}, \boldsymbol{\Delta}_{\mathbf{1}}, \boldsymbol{\Gamma}_{\mathbf{1}}\right)=\rho(\mathbf{W}, \mathbf{Y})$, then it means that $\rho_{A}\left(\mathbf{W}, \boldsymbol{\Gamma}_{\mathbf{1}}, \boldsymbol{\Delta}_{\mathbf{1}}, \boldsymbol{\Gamma}_{\mathbf{1}}\right) \leq$ $g \_r a n k\left(C B_{1}\right)$. As, by definition, $\rho_{A}\left(\mathbf{W}, \boldsymbol{\Gamma}_{\mathbf{1}}, \boldsymbol{\Delta}_{\mathbf{1}}, \boldsymbol{\Gamma}_{\mathbf{1}}\right)=g \_r a n k\left(B_{1}\right)$ we have $g \_r a n k\left(B_{1}\right) \leq g \_r a n k\left(C B_{1}\right)$, which implies that $g_{-} \operatorname{rank}\left(B_{1}\right)=g_{-} \operatorname{rank}\left(C B_{1}\right)$. So, $g_{-} \operatorname{dim}\left(\operatorname{Im}\left(B_{1}\right) \cap \operatorname{ker} C\right)=0$ and consequently $\mathcal{R}_{B_{1}}=\operatorname{Im}\left(B_{1}\right)$. Using Statement St1. proved above, we have then that $g_{-} \operatorname{dim}\left(C \mathcal{R}_{H}\right)=g_{-} \operatorname{dim}\left(C \mathcal{R}_{B_{1}}\right)=g_{-} \operatorname{rank}\left(C B_{1}\right)=g_{-} \operatorname{rank}\left(B_{1}\right)=$ $\rho_{A}\left(\mathbf{W}, \boldsymbol{\Gamma}_{\mathbf{1}}, \boldsymbol{\Delta}_{\mathbf{1}}, \Gamma_{1}\right)=\rho(\mathbf{W}, \mathbf{Y})$.

Statement $\mathbf{S t} \mathbf{2}$ of the previous lemma allows to compute $g_{-} \operatorname{dim}\left(C \mathcal{R}_{H}\right)$ in the specific case where $\rho_{A}\left(\mathbf{W}, \boldsymbol{\Gamma}_{\mathbf{1}}, \boldsymbol{\Delta}_{\mathbf{1}}, \boldsymbol{\Gamma}_{\mathbf{1}}\right)=$ $\rho(\mathbf{W}, \mathbf{Y})$. Knowing that, by construction, $\rho_{A}\left(\mathbf{W}, \boldsymbol{\Gamma}_{\mathbf{1}}, \boldsymbol{\Delta}_{\mathbf{1}}, \boldsymbol{\Gamma}_{\mathbf{1}}\right) \geq \rho(\mathbf{W}, \mathbf{Y})$, we are interested, in the following paragraph, in the determination of $g_{-} \operatorname{dim}\left(C \mathcal{R}_{H}\right)$ in the case where $\rho_{A}\left(\mathbf{W}, \boldsymbol{\Gamma}_{\mathbf{1}}, \boldsymbol{\Delta}_{\mathbf{1}}, \boldsymbol{\Gamma}_{\mathbf{1}}\right)>\rho(\mathbf{W}, \mathbf{Y})$.

In order to compute exactly $g_{-} \operatorname{dim}\left(C \mathcal{R}_{H}\right)$, we introduce now following subsets $\boldsymbol{\Gamma}_{\mathbf{i}}, \boldsymbol{\Delta}_{\mathbf{i}}$ and $\boldsymbol{\Omega}_{\mathbf{i}}, i>1$, which are the generalisation of $\boldsymbol{\Gamma}_{\mathbf{1}}, \Delta_{\mathbf{1}}$ and $\boldsymbol{\Omega}_{\mathbf{1}}$ introduced above. More precisely, the latter is quite general in order to be applied also in the multiple fault case. It allows us to characterize $g_{-} \operatorname{dim}\left(C \mathcal{R}_{H_{0}}\right)$, where matrix $H_{0}$ is constituted by any 
columns of $H$ and $E$ i.e. $H_{0}=\left(H_{i_{1}}, H_{i_{2}}, \ldots, H_{i_{k}}, E_{j_{1}}, E_{j_{2}}, \ldots, E_{j_{s}}\right)$, where $H_{i}$ and $E_{j}$ represent respectively the $i^{\text {th }}$ column of matrix $H$ and the $j^{\text {th }}$ column of matrix $E$. To such matrix, we associate vertex subset $\mathbf{V}_{\mathbf{0}} \subseteq \mathbf{W} \cup \mathbf{F}$ such that $\mathbf{V}_{\mathbf{0}}=\left\{\mathbf{w}_{\mathbf{i}_{1}}, \mathbf{w}_{\mathbf{i}_{2}}, \ldots, \mathbf{w}_{\mathbf{i}_{\mathbf{k}}}, \mathbf{f}_{\mathbf{j}_{1}}, \mathbf{f}_{\mathbf{j}_{2}}, \ldots, \mathbf{f}_{\mathbf{j}_{\mathrm{s}}}\right\}$.

Algorithm 1 Consider any $\mathbf{V}_{\mathbf{0}} \subseteq \mathbf{W} \cup \mathbf{F}$.

\section{Initialisation:}

$\leftrightarrow \mathbf{\Omega}_{\mathbf{0}}=\mathcal{M}\left(H_{0}\right), \mathbf{J}_{0}=\mathbf{X}, i=0$

\section{Step $i$ :}

$\hookrightarrow \rho_{i}=\max _{\gamma \in \mathbf{\Omega}_{\mathbf{i}}} \rho(\gamma, \mathbf{Y}), \mu_{i}=\min _{\gamma \in \mathbf{\Omega}_{\mathbf{i}}, \rho(\gamma, \mathbf{Y})=\rho_{i}} \mu(\gamma, \mathbf{Y})$,

$\leftrightarrow \mathbf{J}_{\mathbf{i}+1} \stackrel{\text { def }}{=}\left\{\mathbf{x}_{\mathbf{j}} \in \mathbf{J}_{\mathbf{i}}, \max _{\gamma \in \mathbf{\Omega}_{\mathbf{i}}} \rho\left(\gamma, \mathbf{Y} \cup\left\{\mathbf{x}_{\mathbf{j}}\right\}\right)=\rho_{i}\right.$ and $\left.\min _{\gamma \in \mathbf{\Omega}_{\mathbf{i}}, \rho\left(\gamma, \mathbf{Y} \cup\left\{\mathbf{x}_{\mathbf{j}}\right\}\right)=\rho_{i}} \mu\left(\gamma, \mathbf{Y} \cup\left\{\mathbf{x}_{\mathbf{j}}\right\}\right)=\mu_{i}\right\}$,

$\leftrightarrow \boldsymbol{\Delta}_{\mathbf{i}+\mathbf{1}} \stackrel{\text { def }}{=} \boldsymbol{\Delta}_{\mathbf{i}} \cup\left\{\mathbf{x}_{\mathbf{j}} \in \mathbf{X} \backslash \mathbf{J}_{\mathbf{i}+\mathbf{1}}, \max _{\gamma \in \mathbf{\Omega}_{\mathbf{i}}} \rho\left(\gamma, \mathbf{Y} \cup\left\{\mathbf{x}_{\mathbf{j}}\right\}\right)>\rho_{i}\right.$ or $\left.\min _{\gamma \in \mathbf{\Omega}_{\mathbf{i}}, \rho\left(\gamma, \mathbf{Y} \cup\left\{\mathbf{x}_{\mathbf{j}}\right\}\right)=\rho_{i}} \mu\left(\gamma, \mathbf{Y} \cup\left\{\mathbf{x}_{\mathbf{j}}\right\}\right)<\mu_{i}-1\right\}$,

$\rightarrow \boldsymbol{\Gamma}_{\mathbf{i}+\mathbf{1}} \stackrel{\text { def }}{=} \mathbf{X} \backslash\left(\mathbf{J}_{\mathbf{i}+\mathbf{1}} \cup \boldsymbol{\Delta}_{\mathbf{i}+\mathbf{1}}\right)$,

$\leftrightarrow \boldsymbol{\Gamma}_{\mathbf{i n t}} \stackrel{\text { def }}{=}\left\{\mathbf{x}_{\mathbf{i}} \in \boldsymbol{\Gamma}_{\mathbf{i}+\mathbf{1}}, \exists \mathbf{W}-\boldsymbol{\Gamma}_{\mathbf{i}+\mathbf{1}}\right.$ paths $P$ and $Q$ included in $\boldsymbol{\Delta}_{\mathbf{i}+\mathbf{1}}$ such that $\alpha(Q)=\mathbf{x}_{\mathbf{i}}$ and $P$ is a strict multiple of Q $\}$,

$\Gamma_{\text {ext }}=\Gamma_{1} \backslash \Gamma_{\text {int }}$,

$\leftrightarrow$ Compute $\rho_{A}\left(\mathbf{V}_{\mathbf{0}}, \boldsymbol{\Gamma}_{\mathbf{i}+\mathbf{1}}, \boldsymbol{\Delta}_{\mathbf{i}+\mathbf{1}}, \boldsymbol{\Gamma}_{\mathbf{i}+\mathbf{1}}\right)$ by substituting $\boldsymbol{\Delta}_{\mathbf{1}}$ and $\boldsymbol{\Gamma}_{\mathbf{1}}$ in Definition 4.2 by $\boldsymbol{\Delta}_{\mathbf{i}+\mathbf{1}}$ and $\boldsymbol{\Gamma}_{\mathbf{i}+\mathbf{1}}$ respectively,

$\leftrightarrow$ If $\rho_{A}\left(\mathbf{V}_{\mathbf{0}}, \boldsymbol{\Gamma}_{\mathbf{i}+\mathbf{1}}, \boldsymbol{\Delta}_{\mathbf{i}+\mathbf{1}}, \boldsymbol{\Gamma}_{\mathbf{i}+\mathbf{1}}\right)>\rho_{i}$, then

$\boldsymbol{\Omega}_{\mathbf{i}+\mathbf{1}} \stackrel{\text { def }}{=}\left\{\gamma \subseteq \boldsymbol{\Gamma}_{\mathbf{i}}, \operatorname{card}(\gamma)=\rho_{A}\left(\mathbf{V}_{\mathbf{0}}, \gamma, \boldsymbol{\Delta}_{\mathbf{i}+\mathbf{1}}, \boldsymbol{\Gamma}_{\mathbf{i}+\mathbf{1}}\right)=\rho_{A}\left(\mathbf{V}_{\mathbf{0}}, \boldsymbol{\Gamma}_{\mathbf{i}+\mathbf{1}}, \boldsymbol{\Delta}_{\mathbf{i}+\mathbf{1}}, \boldsymbol{\Gamma}_{\mathbf{i}+\mathbf{1}}\right)\right\}, \mathrm{i}=\mathrm{i}+1$, go to Step $\boldsymbol{i}$

$\leftrightarrow$ Else $\boldsymbol{\Gamma}_{*}\left(\mathbf{V}_{\mathbf{0}}\right)=\boldsymbol{\Gamma}_{\mathbf{i}+\mathbf{1}}, \boldsymbol{\Delta}_{*}\left(\mathbf{V}_{\mathbf{0}}\right)=\boldsymbol{\Delta}_{\mathbf{i}+\mathbf{1}}, \underline{\rho_{*}}\left(\mathbf{V}_{\mathbf{0}}\right)=\rho_{\mathbf{i}}, \boldsymbol{\Omega}_{*}\left(\mathbf{V}_{\mathbf{0}}\right)=\boldsymbol{\Omega}_{\mathbf{i}}$

Example 4.6 Let us illustrate Algorithm 1 and Definition 4.2 and compute $\rho_{*}(\mathbf{W})$ for the structured system associated to the digraph of Figure 2.

\section{Initialisation:}

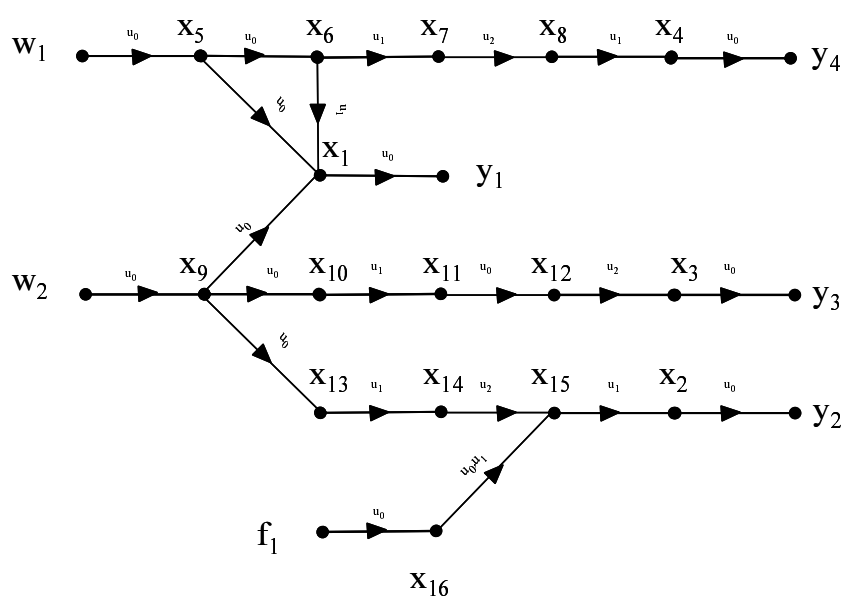

Figure 2.: Example 4.6

$\leftrightarrow \mathbf{\Omega}_{\mathbf{0}}=\left\{\left\{\mathbf{x}_{\mathbf{5}}, \mathbf{x}_{\mathbf{9}}\right\}\right\}, \mathbf{J}_{0}=\mathbf{X}, i=0$ 


\section{Step 0:}

$\leftrightarrow \rho_{0}=\rho\left(\left\{\mathbf{x}_{\mathbf{5}}, \mathbf{x}_{\mathbf{9}}\right\}, \mathbf{Y}\right)=2, \mu_{0}=\mu\left(\left\{\mathbf{x}_{\mathbf{5}}, \mathbf{x}_{\mathbf{9}}\right\}, \mathbf{Y}\right)=9$,

$\rightarrow \mathbf{J}_{\mathbf{1}}=\left\{\mathbf{x}_{16}\right\}$,

$\rightarrow \Delta_{1}=\left\{\mathbf{x}_{5}, \mathbf{x}_{6}, \mathbf{x}_{7}, \mathbf{x}_{8}, \mathbf{x}_{9}, \mathbf{x}_{10}, \mathbf{x}_{11}, \mathbf{x}_{12}, \mathbf{x}_{13}, \mathbf{x}_{14}, \mathbf{x}_{15}\right\}$,

$\leftrightarrow \Gamma_{1}=\left\{\mathbf{x}_{1}, \mathbf{x}_{2}, \mathbf{x}_{3}, \mathbf{x}_{4}\right\}$,

We have also that $\boldsymbol{\Gamma}_{\text {int }}=\left\{\mathbf{x}_{\mathbf{1}}\right\}$.

$\leftrightarrow$ Computation of $\rho_{A}\left(\mathbf{W}, \boldsymbol{\Gamma}_{\mathbf{1}}, \boldsymbol{\Delta}_{\mathbf{1}}, \boldsymbol{\Gamma}_{\mathbf{1}}\right)$ : First note that $\rho_{A}\left(\mathbf{W}, \boldsymbol{\Gamma}_{\mathbf{1}}, \boldsymbol{\Delta}_{\mathbf{1}}, \boldsymbol{\Gamma}_{\mathbf{1}}\right)=4$. Indeed, there exist three $A$-disjoint linkings of size 4: $\mathcal{L}_{1}=\left\{P_{1}, P_{2}, P_{3}, P_{4}\right\}$ and $\mathcal{L}_{2}=\left\{P_{5}, P_{2}, P_{3}, P_{4}\right\}, \mathcal{L}_{3}=\left\{P_{6}, P_{2}, P_{3}, P_{4}\right\}$, where: $P_{1}=\mathbf{w}_{1} \stackrel{u_{0}}{\longrightarrow}$ $\mathbf{x}_{\mathbf{5}} \stackrel{u_{0}}{\longrightarrow} \mathbf{x}_{\mathbf{1}} \stackrel{\text { def }}{=} Q_{1}, P_{2}=\mathbf{w}_{\mathbf{1}} \stackrel{u_{0}}{\longrightarrow} \mathbf{x}_{\mathbf{5}} \stackrel{u_{0}}{\longrightarrow} \mathbf{x}_{\mathbf{6}} \stackrel{u_{1}}{\longrightarrow} \mathbf{x}_{\mathbf{7}} \stackrel{u_{2}}{\longrightarrow} \mathbf{x}_{\mathbf{8}} \stackrel{u_{1}}{\longrightarrow} \mathbf{x}_{\mathbf{4}}, P_{3}=\mathbf{w}_{\mathbf{2}} \stackrel{u_{0}}{\longrightarrow} \mathbf{x}_{\mathbf{9}} \stackrel{u_{0}}{\longrightarrow} \mathbf{x}_{\mathbf{1 0}} \stackrel{u_{1}}{\longrightarrow}$ $\mathbf{x}_{11} \stackrel{u_{0}}{\longrightarrow} \mathbf{x}_{12} \stackrel{u_{2}}{\longrightarrow} \mathbf{x}_{\mathbf{3}}, P_{4}=\mathbf{w}_{\mathbf{2}} \stackrel{u_{0}}{\longrightarrow} \mathbf{x}_{\mathbf{9}} \stackrel{u_{0}}{\longrightarrow} \mathbf{x}_{13} \stackrel{u_{1}}{\longrightarrow} \mathbf{x}_{14} \stackrel{u_{2}}{\longrightarrow} \mathbf{x}_{15} \stackrel{u_{1}}{\longrightarrow} \mathbf{x}_{\mathbf{2}}, P_{5}=\mathbf{w}_{\mathbf{2}} \stackrel{u_{0}}{\longrightarrow} \mathbf{x}_{\mathbf{9}} \stackrel{u_{0}}{\longrightarrow} \mathbf{x}_{\mathbf{1}} \stackrel{\text { def }}{=} Q_{2}$, $P_{6}=\mathbf{w}_{\mathbf{1}} \stackrel{u_{0}}{\longrightarrow} \mathbf{x}_{\mathbf{5}} \stackrel{u_{0}}{\longrightarrow} \mathbf{x}_{\mathbf{6}} \stackrel{u_{1}}{\longrightarrow} \mathbf{x}_{\mathbf{1}}$.

All paths $P_{2}, P_{3}$ and $P_{4}$ are multiple of some prime $\mathbf{W}-\boldsymbol{\Gamma}_{\text {int }}$ paths i.e. $P_{6} \equiv Q_{1} \odot u_{1}, P_{2} \equiv Q_{1} \odot\left(u_{1} \cdot u_{2} \cdot u_{1}\right) \equiv P_{6} \odot\left(u_{2} \cdot u_{1}\right)$, $P_{3} \equiv Q_{2} \odot\left(u_{1} \cdot u_{0} \cdot u_{2}\right)$ and $P_{4} \equiv Q_{2} \odot\left(u_{1} \cdot u_{2} \cdot u_{1}\right)$. To these paths we can associate the following complete families: $S\left(P_{1}\right)=\emptyset, S\left(P_{2}\right)=\left\{Q_{1} \odot\left(u_{1} \cdot u_{2} \cdot u_{1}\right), Q_{1} \odot\left(u_{1} \cdot u_{1} \cdot u_{2} \cdot u_{1}\right), Q_{2} \odot\left(u_{1} \cdot u_{1} \cdot u_{2} \cdot u_{1}\right), Q_{1} \odot\left(u_{2} \cdot u_{1}\right)\right\}$, $S\left(P_{3}\right)=\left\{Q_{2} \odot\left(u_{1} \cdot u_{0} \cdot u_{2}\right), Q_{1} \odot\left(u_{1} \cdot u_{0} \cdot u_{2}\right), Q_{1} \odot\left(u_{0} \cdot u_{2}\right)\right\}, S\left(P_{4}\right)=\left\{Q_{2} \odot\left(u_{1} \cdot u_{2} \cdot u_{1}\right), Q_{1} \odot\left(u_{1} \cdot u_{2} \cdot u_{1}\right), Q_{1} \odot\left(u_{2} \cdot u_{1}\right)\right\}$ or $S\left(P_{4}\right)=\left\{Q_{2} \odot\left(u_{1} \cdot u_{2} \cdot u_{1}\right), Q_{1} \odot\left(u_{1} \cdot u_{1} \cdot u_{2} \cdot u_{1}\right), Q_{2} \odot\left(u_{1} \cdot u_{1} \cdot u_{2} \cdot u_{1}\right), Q_{1} \odot\left(u_{2} \cdot u_{1}\right)\right\}$. This last family is more suitable because it allows to obtain a set satisfying constraint Ca of Definition 4.2 (because $P_{2} \equiv Q_{1} \odot\left(u_{1} \cdot u_{2} \cdot u_{1}\right)$ ). Therefore, $\rho_{A}\left(\mathbf{W}, \boldsymbol{\Gamma}_{\mathbf{1}}, \boldsymbol{\Delta}_{\mathbf{1}}, \boldsymbol{\Gamma}_{\mathbf{1}}\right)=4$ and so $\boldsymbol{\Omega}_{\mathbf{1}}=\left\{\left\{\mathbf{x}_{\mathbf{1}}, \mathbf{x}_{\mathbf{2}}, \mathbf{x}_{\mathbf{3}}, \mathbf{x}_{\mathbf{4}}\right\}\right\}$.

$i=1$.

\section{Step 1:}

$\leftrightarrow \rho_{1}=\rho\left(\left\{\mathbf{x}_{1}, \mathbf{x}_{2}, \mathbf{x}_{3}, \mathbf{x}_{\mathbf{4}}\right\}, \mathbf{Y}\right)=4, \mu_{1}=\mu\left(\left\{\mathbf{x}_{1}, \mathbf{x}_{2}, \mathbf{x}_{3}, \mathbf{x}_{4}\right\}, \mathbf{Y}\right)=8$,

$\rightarrow \mathbf{J}_{2}=\left\{\mathbf{x}_{16}\right\}$,

$\rightarrow \Delta_{2}=\left\{\mathbf{x}_{5}, \mathbf{x}_{6}, \mathbf{x}_{7}, \mathbf{x}_{8}, \mathbf{x}_{9}, \mathbf{x}_{10}, \mathbf{x}_{11}, \mathbf{x}_{12}, \mathbf{x}_{13}, \mathbf{x}_{14}, \mathbf{x}_{15}\right\}$,

$\leftrightarrow \Gamma_{2}=\left\{\mathbf{x}_{1}, \mathbf{x}_{2}, \mathbf{x}_{3}, \mathbf{x}_{4}\right\}$,

We have also that $\boldsymbol{\Gamma}_{\text {int }}=\left\{\mathbf{x}_{\mathbf{1}}\right\}$.

$\leftrightarrow$ Since $\boldsymbol{\Delta}_{\mathbf{2}}=\boldsymbol{\Delta}_{\mathbf{1}}$ and $\boldsymbol{\Gamma}_{\mathbf{2}}=\boldsymbol{\Gamma}_{\mathbf{1}}$ then $\rho_{A}\left(\mathbf{W}, \boldsymbol{\Gamma}_{\mathbf{2}}, \boldsymbol{\Delta}_{\mathbf{2}}, \boldsymbol{\Gamma}_{\mathbf{2}}\right)=\rho_{A}\left(\mathbf{W}, \boldsymbol{\Gamma}_{\mathbf{1}}, \boldsymbol{\Delta}_{\mathbf{1}}, \boldsymbol{\Gamma}_{\mathbf{1}}\right)=4$.

As, $\rho_{A}\left(\mathbf{W}, \boldsymbol{\Gamma}_{\mathbf{2}}, \boldsymbol{\Delta}_{\mathbf{2}}, \boldsymbol{\Gamma}_{\mathbf{2}}\right)=\rho_{1}$, we have that $\boldsymbol{\Gamma}_{*}(\mathbf{W})=\boldsymbol{\Gamma}_{\mathbf{2}}, \boldsymbol{\Delta}_{*}(\mathbf{W})=\boldsymbol{\Delta}_{\mathbf{2}}, \rho_{*}(\mathbf{W})=4, \boldsymbol{\Omega}_{*}(\mathbf{W})=\boldsymbol{\Omega}_{\mathbf{1}}$.

If the edge $\left(\mathbf{x}_{11}, \mathbf{x}_{12}\right)$ was included in $A_{1}$-edges and not in $A_{0}$-edges, neither $S\left(P_{2}\right)=\left\{Q_{1} \odot\right.$ $\left.\left(u_{1} \cdot u_{2} \cdot u_{1}\right), Q_{1} \odot\left(u_{1} \cdot u_{1} \cdot u_{2} \cdot u_{1}\right), Q_{2} \odot\left(u_{1} \cdot u_{1} \cdot u_{2} \cdot u_{1}\right), Q_{1} \odot\left(u_{2} \cdot u_{1}\right)\right\}$ nor $S\left(P_{4}\right)=$ $\left\{Q_{2} \odot\left(u_{1} \cdot u_{2} \cdot u_{1}\right), Q_{1} \odot\left(u_{1} \cdot u_{1} \cdot u_{2} \cdot u_{1}\right), Q_{2} \odot\left(u_{1} \cdot u_{1} \cdot u_{2} \cdot u_{1}\right), Q_{1} \odot\left(u_{2} \cdot u_{1}\right)\right\}$ are permitted since they contain an element $Q_{2} \odot\left(u_{1} \cdot u_{1} \cdot u_{2} \cdot u_{1}\right)$ which is multiple of $P_{3}$ i.e. $Q_{2} \odot\left(u_{1} \cdot u_{1} \cdot u_{2} \cdot u_{1}\right) \equiv P_{3} \odot u_{1}$ and so constraint $\mathbf{C b}$ is not satisfied. In this case, we must take other families: $S\left(P_{2}\right)=\left\{Q_{1} \odot\left(u_{1} \cdot u_{2} \cdot u_{1}\right), Q_{1} \odot\left(u_{1} \cdot u_{1} \cdot u_{2}\right.\right.$. $\left.\left.u_{1}\right), Q_{1} \odot\left(u_{1} \cdot u_{1} \cdot u_{1} \cdot u_{2} \cdot u_{1}\right), Q_{2} \odot\left(u_{1} \cdot u_{1} \cdot u_{1} \cdot u_{2} \cdot u_{1}\right), Q_{1} \odot\left(u_{2} \cdot u_{1}\right)\right\}$ and $S\left(P_{4}\right)=\left\{Q_{2} \odot\left(u_{1} \cdot u_{2} \cdot u_{1}\right), Q_{1} \odot\left(u_{1} \cdot\right.\right.$ $\left.\left.u_{1} \cdot u_{2} \cdot u_{1}\right), Q_{1} \odot\left(u_{1} \cdot u_{1} \cdot u_{1} \cdot u_{2} \cdot u_{1}\right), Q_{2} \odot\left(u_{1} \cdot u_{1} \cdot u_{1} \cdot u_{2} \cdot u_{1}\right), Q_{1} \odot\left(u_{2} \cdot u_{1}\right)\right\}$.

$\rho_{A}\left(\mathbf{W}, \boldsymbol{\Gamma}_{\mathbf{1}}, \boldsymbol{\Delta}_{\mathbf{1}}, \boldsymbol{\Gamma}_{\mathbf{1}}\right)=4$ and $\boldsymbol{\Omega}_{\mathbf{1}}=\left\{\left\{\mathbf{x}_{\mathbf{1}}, \mathbf{x}_{\mathbf{2}}, \mathbf{x}_{\mathbf{3}}, \mathbf{x}_{\mathbf{4}}\right\}\right\}$. Consequently, also in this case, $\rho_{*}(\mathbf{W})=4$.

The following lemma characterizes the value of $g_{-} \operatorname{dim}\left(C \mathcal{R}_{H}\right)$ :

Lemma 4.7 Consider $\operatorname{SBLS}\left(\Sigma_{\Lambda}\right)$ represented by digraph $\mathcal{G}\left(\Sigma_{\Lambda}\right)$. We have 


$$
g_{-} \operatorname{dim}\left(C \mathcal{R}_{H}\right)=\rho_{*}(\mathbf{W})
$$

\section{Proof:}

Let $\kappa$ be the first integer such that $\rho_{A}\left(\mathbf{W}, \boldsymbol{\Gamma}_{\kappa+\mathbf{1}}, \boldsymbol{\Delta}_{\kappa+\mathbf{1}}, \boldsymbol{\Gamma}_{\kappa+\mathbf{1}}\right)=\rho_{\kappa}=\max _{\gamma \in \mathbf{\Omega}_{k}} \rho(\gamma, \mathbf{Y})$.

From Statement St1 of Corollary 4.5, we can write that $g_{-} \operatorname{dim}\left(C \mathcal{R}_{H}\right)=g_{-} \operatorname{dim}\left(C \mathcal{R}_{B_{1}}\right)$ for almost all structured matrix $B_{1}$ such that $\mathcal{M}\left(B_{1}\right)$ is equal to $\boldsymbol{\Omega}_{\mathbf{1}}$. Moreover, if we denote $\mathbf{W}^{\prime}=\left\{\mathbf{w}_{\mathbf{1}}^{\prime}, \ldots, \mathbf{w}_{\mathbf{d}^{\prime}}^{\prime}\right\}$, with $d^{\prime}=$ $\rho_{A}\left(\mathbf{W}, \boldsymbol{\Gamma}_{\mathbf{i}-\mathbf{1}}, \boldsymbol{\Delta}_{\mathbf{i}-\mathbf{1}}, \boldsymbol{\Gamma}_{\mathbf{i}-\mathbf{1}}\right)$, a virtual vertex subset connected to vertex subset $\mathbf{X}$ through the virtual edges noted $B_{i}$-edges $=\left\{\left(\mathbf{w}_{\mathbf{k}}^{\prime}, \mathbf{x}_{\mathbf{j}}\right) \mid B_{i}(j, k) \neq 0\right\}$, we have that $\rho_{A}\left(\mathbf{W}, \boldsymbol{\Gamma}_{\mathbf{i}}, \boldsymbol{\Delta}_{\mathbf{i}}, \boldsymbol{\Gamma}_{\mathbf{i}}\right)=\rho_{A}\left(\mathbf{W}^{\prime}, \boldsymbol{\Gamma}_{\mathbf{i}}, \boldsymbol{\Delta}_{\mathbf{i}}, \boldsymbol{\Gamma}_{\mathbf{i}}\right)$. Thus, we can generalize Statement St1 of Corollary 4.5, and so we have iteratively, $g_{-} \operatorname{dim}\left(C \mathcal{R}_{H}\right)=g_{-} \operatorname{dim}\left(C \mathcal{R}_{B_{1}}\right)=g_{-} \operatorname{dim}\left(C \mathcal{R}_{B_{2}}\right)=\ldots=$ $g_{-} \operatorname{dim}\left(C \mathcal{R}_{B_{k}}\right)$, for almost all structured matrix $B_{i}, 1 \leq i \leq \kappa+1$, such that $\mathcal{M}\left(e_{\Gamma_{i}} B_{i}\right)$ is equal to $\boldsymbol{\Omega}_{\mathbf{i}}$.

Moreover, as $\rho_{A}\left(\mathbf{W}, \boldsymbol{\Gamma}_{\kappa+\mathbf{1}}, \boldsymbol{\Delta}_{\kappa+\mathbf{1}}, \boldsymbol{\Gamma}_{\kappa+\mathbf{1}}\right)=\rho_{\kappa}=\max _{\gamma \in \boldsymbol{\Omega}_{\kappa}}(\rho(\gamma, \mathbf{Y}))$, statement $\mathbf{S t 2}$ applied to $\boldsymbol{\Omega}_{\kappa}$ gives $g_{-} \operatorname{dim}\left(C \mathcal{R}_{B_{k}}\right)=$ $\rho_{A}\left(\mathbf{W}, \boldsymbol{\Gamma}_{\kappa+1}, \boldsymbol{\Delta}_{\kappa+1}, \boldsymbol{\Gamma}_{\kappa+1}\right)=\rho_{*}(\mathbf{W})$. Thus, $g_{-} \operatorname{dim}\left(C \mathcal{R}_{H}\right)=\rho_{*}(\mathbf{W})$ and the Lemma is proved.

Proposition 4.8 Consider structured system $\left(\Sigma_{\Lambda}\right)$ represented by digraph $\mathcal{G}\left(\Sigma_{\Lambda}\right)$. In the single fault case, the BFPRG has generically a solution iff $\rho_{*}(\mathbf{W} \cup \mathbf{F})>\rho_{*}(\mathbf{W})$.

\section{Proof:}

The proof is immediate according to Lemma 4.7 and to Lemma 2.1.

Note, finally, that for SBLS $\left(\Sigma_{\Lambda}\right)$, it is quite easy to prove that $\mathcal{R}_{H}^{f}=\operatorname{span}\left\{e_{j}, \mathbf{x}_{\mathbf{j}} \in \boldsymbol{\Gamma}_{*}(\mathbf{W}) \cup \boldsymbol{\Delta}_{*}(\mathbf{W})\right\}$, where $e_{j}$ is the $j^{\text {th }}$ Euclidean vector.

For the system described in Example 4.6, $\rho_{*}\left(\mathbf{W} \cup\left\{\mathbf{f}_{\mathbf{1}}\right\}\right)=4=\rho_{*}(\mathbf{W})$ and so the BFPRG is not generically solvable.

\subsection{Problem of Residual Generation: Multiple fault case}

4.2.1 Bilinear Fundamental Problem of Residual Generation with eventually simultaneous failures events. In the case of multiple and possibly simultaneous failure events we must solve the BFPRG for each failure component $f_{i}$ for $i=1, \ldots, q$. Thus, directly from Proposition 4.8 we can enounce:

COROLLARY 4.9 Consider structured system $\left(\Sigma_{\Lambda}\right)$ represented by digraph $\mathcal{G}\left(\Sigma_{\Lambda}\right)$. The BFPRG with multiple and simultaneous failure events has generically a solution iff

$$
\forall i \in\{1,2, \ldots, q\}, \quad \rho_{*}(\mathbf{W} \cup \mathbf{F})>\rho_{*}\left(\mathbf{W} \cup \mathbf{F} \backslash\left\{\mathbf{f}_{\mathbf{i}}\right\}\right)
$$

4.2.2 Problem of Residual Generation with no simultaneous faults. In the case where only one failure can occur at a time on the system, we have:

Corollary 4.10 Consider structured system $\left(\Sigma_{\Lambda}\right)$ represented by digraph $\mathcal{G}\left(\Sigma_{\Lambda}\right)$ and assume that only one failure event can occur at a time on the system. The BFPRG has generically a solution iff

$$
\forall i, j \in\{1, \ldots, q\}, \text { with } i \neq j, \quad \rho_{*}\left(\mathbf{W} \cup\left\{\mathbf{f}_{\mathbf{i}}, \mathbf{f}_{\mathbf{j}}\right\}\right)>\rho_{*}\left(\mathbf{W} \cup\left\{\mathbf{f}_{\mathbf{j}}\right\}\right)
$$




\section{Computational aspects and concluding remarks}

Let us analyse briefly the computational aspects of the proposed method. Mainly, according to Algorithm 1, there are three stages in the computation of $\rho_{*}\left(\mathbf{V}_{\mathbf{0}}\right), \mathbf{V}_{\mathbf{0}} \subseteq \mathbf{W} \cup \mathbf{F}$ :

- Computation of $\rho(\gamma, \mathbf{Y})$ and $\mu(\gamma, \mathbf{Y})$ : we use classical algorithms of maximum flow calculation, the overall complexity is equal to $O\left(N^{2} \sqrt{M}\right)$, where $M=(m+1) n^{2}+n(q+d)+p$ is the number of edges and $N=n+q+p+d$ is the number of vertices in the digraph.

- Extraction of the maximal $A$-disjoint linkings: on the basis of the precedent computation, we add to the disjoint paths (which are $A$-disjoint) other paths to complete the linking. There are two stages: path extraction and verification of conditions Cond1 and Cond2. The overall complexity is equal to $O\left(N^{3} M \sqrt{M}\right)$.

- Association of complete families to each path and computation of $\boldsymbol{\Omega}_{\mathrm{i}}$ : these two calculations are made simultaneously, using logical expressions to characterize $\boldsymbol{\Omega}_{\mathbf{i}}$. The construction of complete families is done according to constraints $\mathbf{C a}$ and $\mathbf{C b}$ of Definition 4.2. The overall complexity of this step is estimated equal to $O\left(N^{3} M\right)$.

Note that at step $i+1$, we use results of step $i$, the definition of $\boldsymbol{\Delta}_{\mathbf{i}}$ and $\boldsymbol{\Gamma}_{\mathbf{i}}$ is very useful to reduce the number of paths to be tested in the algorithm. Moreover, at each step, we construct a structured matrix $\bar{B}_{i}$ such that $\mathcal{M}\left(\bar{B}_{i}\right)=\mathbf{\Omega}_{\mathbf{i}}$, this allows also to reduce considerably the number of paths to be tested in the algorithm.

Consequently, if we assume, without loss of generality, that $p \leq n, q \leq n$ and $d \leq n$ the overall complexity of Algorithm 1 is then $O\left(m n^{5} \sqrt{n m}\right)$.

To summarize, in this paper, we propose a graph-theoretic approach to provide necessary and sufficient condition for the generic solvability of BFPRG for structured bilinear systems. The proposed method needs few information about the system and is free from numerical difficulties. This makes it well-suited for large scale and uncertain systems and also in conception stages.

Finally, the use of graph-theoretic tools makes easy the visualization of the system structure. This may be helpful for the optimisation of sensor placement to fulfill the solvability of residual generation problems as it is done in (Commault and Dion 2003) for structured linear systems.

\section{References}

M. Blanke, M. Kinnaert, J. Lunze and M. Staroswiecki, Diagnosis and Fault-Tolerant Control, Heidelberg: Springer-Verlag, 2003.

C. Commault and J.M. Dion, "Sensor Location for Diagnosis in Linear Structured Systems with Disturbances", in $42^{\text {nd }}$ IEEE Conference on Decision and Control, Hawaii, U.S.A., 2003.

C. Commault, J.M. Dion and V. Hovelaque, "A Geometric Approach for Structured Systems: Application to Disturbance Decoupling”, Automatica, 33(3), pp. 403-409, 1997.

C. Commault, J.M. Dion, O. Sename and R. Motyeian, "Observer-Based Fault Diagnosis for Structured Systems", IEEE Transactions on Automatic Control, 47(12), pp. 2074-2079, 2002.

C. De Persis and A. Isidori, "On the Observability Codistributions of a Nonlinear System", System $\mathcal{E}$ Control 
Letters, 40(5), pp. 297-304, 2000.

C. De Persis and A. Isidori, "A geometric approach to nonlinear fault detection and isolation", IEEE Transactions on Automatic Control, 46(6), pp. 853-865, 2001.

J.M. Dion, C. Commault and van der J.W. Woude, "Generic Properties and Control of Linear Structured Systems: a Survey", Automatica, 39(7), pp. 1125-1144, 2003.

D. Düstgör, V. Cocquempot, M. Staroswiecki and E. Frisk, "Isolabilité Structurelle Des Défauts. Application À un Modèle de Vanne", RS - JESA, 38(1), pp. 103-123, 2004.

J. Gertler, Fault detection and diagnosis in engineering systems, New York, U.S.A.: Marcel Dekker, 1998.

H. Hammouri, K. Kabore and M. Kinnaert, "A geometric approach to fault detection and isolation for bilinear systems”, IEEE Transactions on Automatic Control, 46(9), pp. 1451-1455, 2001.

H. Hammouri, M. Kinnaert and E.H. El Yaagoubi, "Observer based approach to fault detection and isolation for non linear systems", IEEE Transactions on Automatic Control, 44(10), pp. 1879-1884, 1999.

M. Kinnaert, "Robust Fault Detection Based on Observers for Bilinear Systems.”, Automatica, 35(11), pp. 18291842, 1999.

M.A. Massoumnia, G.C. Verghese and A.S. Willsky, "Failure detection and identification", IEEE Transactions on Automatic Control, 34(3), pp. 316-321, 1989.

K. Murota, System Analysis by Graphs and Matroids, New York, U.S.A.: Springer-Verlag, 1987.

M. Staroswiecki, "Structural Analysis for Fault Detection and Isolation and for Fault Tolerant Control", in: Fault Diagnosis and Fault Tolerant Control., P. Franck and M. Blanke, Eds, Oxford, U.K.: Eolss Publishers, 2002. van der J.W. Woude, "The Generic Number of Invariant Zeros of a Structured Linear System", SIAM Journal of Control and Optimization, 38(1), pp. 1-21, 2000.

H. Yang and M. Saif, "State Observation, Failure Detection and Isolation (FDI) in Bilinear Systems", in Proceedings of the 34th IEEE Conference on Decision and Control, New Orleans, U.S.A., 1995, pp. 2391-2396.

D. Yu and D.N. Shields, “A Bilinear Fault Detection Observer”, Automatica, 32(11), pp. 1597-1602, 1996.

D. Yu, D.N. Shields and S. Daley, "A Bilinear Fault Detection Observer and its Application to a Hydraulic Drive System”, International Journal of Control, 64(6), pp. 1023-1047, 1996. 